\title{
KMOS view of the Galactic Centre - II. Metallicity distribution of late-type stars *
}

\author{
A. Feldmeier-Krause,${ }^{1} \dagger$ W. Kerzendorf, ${ }^{1}$ N. Neumayer, ${ }^{2}$ R. Schödel, ${ }^{3}$ \\ F. Nogueras-Lara ${ }^{3}$ T. Do, ${ }^{4}$ P. T. de Zeeuw,${ }^{1,5}$ and H. Kuntschner ${ }^{1}$ \\ ${ }^{1}$ European Southern Observatory (ESO), Karl-Schwarzschild-Straße 2, D-85748 Garching, Germany \\ ${ }^{2}$ Max-Planck-Institut für Astronomie, Königsstuhl 17, D-69117 Heidelberg, Germany \\ ${ }^{3}$ Instituto de Astrofísica de Andalucía (CSIC), Glorieta de la Astronomía s/n, E-18008 Granada, Spain \\ ${ }^{4}$ UCLA Galactic Center Group, Physics and Astronomy Department, UCLA, Los Angeles, CA 90095-1547, USA \\ ${ }^{5}$ Sterrewacht Leiden, Leiden University, Postbus 9513, NL-2300 RA Leiden, The Netherlands
}

Accepted 2016 September 12. Received 2016 September 9; in original form 2016 May 20

\begin{abstract}
Knowing the metallicity distribution of stars in the Galactic Centre has important implications for the formation history of the Milky Way nuclear star cluster. However, this distribution is not well known, and is currently based on a small sample of fewer than 100 stars. We obtained near-infrared $K$ band spectra of more than 700 late-type stars in the central $4 \mathrm{pc}^{2}$ of the Milky Way nuclear star cluster with the integral-field spectrograph KMOS (VLT). We analyse the medium-resolution spectra using a full-spectral fitting method employing the Göttingen Spectral library of synthetic PHOENIX spectra. The derived stellar metallicities range from metal-rich $[M / H]>+0.3$ dex to metal-poor $[M / H]<-1.0$ dex, with a fraction of $5.2_{-3.1}^{+6.0}$ per cent metal-poor $([M / H] \leqslant-0.5$ dex $)$ stars. The metal-poor stars are distributed over the entire observed field. The origin of metal-poor stars remains unclear. They could originate from infalling globular clusters. For the metal-rich stellar population $([M / H]>0$ dex $)$ a globular cluster origin can be ruled out. As there is only a very low fraction of metal-poor stars in the central $4 \mathrm{pc}^{2}$ of the Galactic Centre, we believe that our data can discard a scenario in which the Milky Way nuclear star cluster is purely formed from infalling globular clusters.
\end{abstract}

Key words: stars: late-type - Galaxy: centre - infrared: stars.

\section{INTRODUCTION}

A dense concentration of stars is located within the central 10 pc of our Galaxy. These stars form the Milky Way's nuclear star cluster. The centre of this star cluster hosts the Milky Way's supermassive black hole, Sgr A*. It is unclear how the nuclear star cluster and the supermassive black hole formed and evolved. Many other galaxies host either a nuclear star cluster, a central supermassive black hole, or both (e.g. Böker et al. 2002; Côté et al. 2006; Seth et al. 2008a; Graham \& Spitler 2009; Neumayer \& Walcher 2012).

Two main formation scenarios for nuclear star clusters have been proposed. The stars may have formed 'in situ', i.e., in the centre of the galaxy where they are located now (e.g. Loose et al. 1982; Schinnerer et al. 2008; Seth et al.

* Based on observations collected at the European Organisation for Astronomical Research in the Southern Hemisphere, Chile (60.A-9450(A)).

† E-mail: afeldmei@uchicago.edu 2008b; Pflamm-Altenburg \& Kroupa 2009). The second possibility is that the stars formed in clusters further out. Star clusters might have migrated to the centre of the galaxy to form the nuclear star cluster (e.g. Tremaine et al. 1975; Capuzzo-Dolcetta \& Miocchi 2008; Agarwal \& Milosavljević 2011; Gnedin et al. 2014; Antonini 2013). In particular, massive globular clusters may have fallen into galactic nuclei through dynamical friction and thus have seeded nuclear star clusters (e.g. Antonini et al. 2012). Studying the stellar populations of the nuclear star cluster will shed light on the formation scenario, possibly distinguishing between the presented scenarios. In particular, the stellar metallicity distribution is useful to infer the formation history of the nuclear star cluster. A narrow distribution of age and metallicity would suggest that all stars formed from the same gas cloud in one single burst of star formation. On the other hand, a spread in age and metallicity would reveal several star formation events, or even continuous star formation. An inhomogeneous distribution of the metallicity over the nuclear star cluster might be produced by the migration of star clusters 
with different intrinsic metallicities from the outskirts to the centre of the galaxy (Perets \& Mastrobuono-Battisti 2014). In particular, if globular clusters have contributed significant mass to a nuclear star cluster, then we would expect to find a considerable fraction of low-metallicity stars in it, as globular cluster stars have typically low metallicities, $[\mathrm{Fe} / \mathrm{H}]$. In the Milky Way globular clusters, $[\mathrm{Fe} / \mathrm{H}]$ ranges from -2.37 to 0.0 dex with a median at $[\mathrm{Fe} / \mathrm{H}]=-1.32$ dex (Harris 1996, 2010 edition). Only about 10 per cent of the Milky Way globular clusters have metallicities $[\mathrm{Fe} / \mathrm{H}]$ greater than $-0.5 \mathrm{dex}$ (Bica et al. 2006).

Due to its proximity, the Milky Way nuclear star cluster is the perfect laboratory to study the composition and structure of a nuclear star cluster. The cluster is at a distance of approximately $8 \mathrm{kpc}$ (Ghez et al. 2008; Gillessen et al. 2009; Chatzopoulos et al. 2015). It has a mass of approximately $2.5 \times 10^{7} \mathrm{M}_{\odot}$ (Schödel et al. 2014a; Feldmeier et al. 2014) and a half-light radius of 4.2 pc (Schödel et al. 2014a). Many of the stars observed in the Galactic Centre are cool late-type stars. They are distributed throughout the cluster (Feldmeier-Krause et al. 2015). Most of the cool stars are red giants that formed several Gyr ago, though some latetype stars are younger supergiant stars (Blum et al. 2003; Pfuhl et al. 2011). Several red giants of intermediate age (50$500 \mathrm{Myr}$ ) have been found as well (Nishiyama et al. 2016). With the exception of short-lived O/B stars and bright Astars, main-sequence stars are too faint to be detected and studied with current instrumentation in the crowded Milky Way centre.

Despite being the arbiter for a number of important questions, there exist only few measurements of iron abundance and metallicity in the Galactic Centre. Lately, the Gaia-ESO Survey and the Apache Point Observatory Galactic Evolution Experiment (APOGEE) measured metallicity and abundances for several thousand stars of the Milky Way (Mikolaitis et al. 2014; Holtzman et al. 2015). However, APOGEE observed only few stars in the Galactic Centre. Measurements of the Gaia-ESO Survey at visual wavelengths are hindered by high extinction $\left(A_{V}=30 \mathrm{mag}\right.$; Scoville et al. 2003) towards the centre of the Milky Way. Infrared spectroscopy is required, preferentially in the near-infrared $K$ band, where extinction decreases to about $A_{K}=2.5 \mathrm{mag}$ (e.g. Clénet et al. 2001; Schödel et al. 2010). Ramírez et al. (2000) and Ryde \& Schultheis (2015) measured the iron abundance of fewer than 20 stars in the Galactic Centre using high-resolution spectroscopy and found a mean iron abundance near solar, $\langle[\mathrm{Fe} / \mathrm{H}]\rangle=+0.1$ dex. Recently, Do et al. (2015) measured the overall metallicity $[M / H]$ on a larger sample of 83 stars and found a large spread, from $[M / H] \lesssim-1.0$ dex to $[M / H] \gtrsim+0.5$ dex. Their sample is concentrated in the central $1 \mathrm{pc}$, where adaptive optics is most useful. Further out, in the inner Galactic bulge, Schultheis et al. (2015) found also evidence for metal-poor $\mathrm{K} / \mathrm{M}$ giants with $[M / H]=-1$ dex. The $\alpha$-elements of these metal-poor stars seem to be enhanced (Schultheis et al. 2015). Concerning metal-rich stars ( $[\mathrm{Fe} / \mathrm{H}>0$ dex) in the Galactic Centre, Cunha et al. (2007) and Ryde \& Schultheis (2015) found enhanced Ca abundances $(0 \mathrm{dex} \lesssim[\mathrm{Ca} / \mathrm{Fe}] \lesssim+0.5 \mathrm{dex})$, while Ryde \& Schultheis (2015) and Ryde et al. (2016) found lower Mg and Si abundances $(-0.2 \lesssim[\alpha / F e] \lesssim+0.2 \mathrm{dex})$.

A systematic metallicity study of a large sample of stars beyond the central $r=0.5 \mathrm{pc}$ of the Milky Way nuclear star cluster has been missing so far. In this study, we present the spectra of more than 700 late-type stars located in an area $>4 \mathrm{pc}^{2}$ of the Galactic Centre. The spectra were obtained with the integral-field spectrograph KMOS (Sharples et al. 2013) in the near-infrared $K$ band. We measure the stellar parameters effective temperature $T_{\text {eff }}$, and overall metallicity $[M / H]$ with full-spectral fitting for more than 700 stars and study their spatial distribution. The outline of this paper is as follows: we present the data set in Section 2. We outline the full-spectral fitting routine and the error estimation in Section 3. In Section 4, we present our results and discuss them in Section 5. The conclusion follows in Section 6 .

\section{DATA SET}

\subsection{Observations and data reduction}

Our spectroscopic observations were performed with KMOS on 2013 September 23, during the KMOS science verification at VLT-UT1 (Antu). We observed an area of $2700 \operatorname{arcsec}^{2}$, which corresponds to approximately $4 \mathrm{pc}^{2}$ at a distance of $8 \mathrm{kpc}$. The field extends over $64.9 \mathrm{arcsec} \times 43.3 \mathrm{arcsec}$, centred on $\alpha=266^{\circ} 4166$ and $\delta=-29^{\circ} .0082$, with a gap of $10.8 \operatorname{arcsec} \times 10.8$ arcsec in the Galactic North-East direction, since one of the 24 KMOS integral-field units (IFUs) was inactive (IFU 13, see fig. 1 of Feldmeier-Krause et al. 2015).

The spectra were taken in the $K$ band $(\sim 19340-24600)$. The KMOS scale is $\sim 2.8 \mathrm{pixel}^{-1}$ in the spectral direction. The spatial scale is $0.2 \operatorname{arcsec}_{\text {pixel }}^{-1} \times 0.2 \operatorname{arcsec}$ pixel $^{-1}$. We observed the field twice with $100 \mathrm{~s}$ exposure time each. For sky subtraction, we made an offset to a dark cloud (G359.94+0.17, $\alpha \approx 266^{\circ} .2, \delta \approx-28^{\circ} 9$; Dutra \& Bica 2001). Further, we observed B dwarfs for telluric corrections. Data reduction was performed with the ESO pipeline and standard recipes for dark correction, flat-fielding, wavelength calibration, and illumination correction. For sky subtraction we used the method by Davies (2007), which is implemented in the pipeline. We removed cosmic rays with the method by van Dokkum (2001). For further details on the data reduction, we refer to Feldmeier-Krause et al. (2015).

We extracted spectra of more than 1000 individual stars with PampelMuse (Kamann et al. 2013). The spectra have a formal signal to noise $>10$. In PAMPELMuse, stars are deblended with a point spread function (PSF) fitting technique. Spectra from stars are also extracted when the stars' PSFs are centred outside of the field of view of the IFU. Therefore, we have more than two exposures for some of the stars.

\subsection{Spectral resolution}

The spectral resolution of KMOS varies spatially for the 24 different IFUs (e.g. Gazak et al. 2015). We measured the line-spread function of each IFU separately on the reconstructed sky data cubes in order to create a resolution map for each IFU. The line-spread function is reasonably well described with a Gaussian, and we fitted three different sky lines in the wavelength region of $\lambda=21900-22400$ for all 
Table 1. Spectral libraries in the $K$ band

\begin{tabular}{lccccc}
\hline Library & $\begin{array}{c}\text { Resolution } \\
(R=\lambda / \Delta \lambda)\end{array}$ & $\begin{array}{c}\text { Spectral } \\
\text { type }\end{array}$ & $\begin{array}{c}\text { Luminosity } \\
\text { class }\end{array}$ & $\begin{array}{c}\text { Spectral region } \\
()\end{array}$ & $\begin{array}{c}\text { Number } \\
\text { of stars }\end{array}$ \\
\hline Wallace \& Hinkle (1996) & $\geqslant 45000$ & F8-M8 & I-V & $20195-23960$ & 9 \\
Wallace \& Hinkle (1997) & 3000 & O4-M7 & I-V & $20202-24096$ & 61 \\
Winge et al. (2009), NIFS v1.5 & 6000 & G8-M3 & I-III & $20700-24700$ & 10 \\
Winge et al. (2009), NIFS v2.0 & 6000 & G8-M3 & I-III & $20200-24300$ & 13 \\
Winge et al. (2009), GNIRS & 18000 & F7-M0 & II-V & $21800-24250$ & 16 \\
\hline
\end{tabular}

23 used IFUs separately. We calculated average resolution maps from four sky exposures for each IFU, and smoothed the maps using a Gaussian with width $\sigma=1$ pix, roughly corresponding to the seeing during the observations. We found that the spectral resolution $R=\lambda / \Delta \lambda$ varies between 3310 and 4660 for the 23 different active IFUs on the three different KMOS detectors. On a single IFU, the spectral resolution has a standard deviation of about $30-150$. The standard deviation of the spectral resolution $R$ over all IFUs is 300 , this is 7 per cent of the mean value. We used the respective $R$ values for fitting the stellar spectra.

\subsection{Data selection}

For this paper we only regard late-type stars, i.e., cool stars with molecular $\mathrm{CO}$ absorption lines at $\lambda \geqslant 22935$. Spectra of early-type stars are presented in Feldmeier-Krause et al. (2015). We visually inspected the spectra and identified 982 stars as late-type stars based on the prominent CO lines.

In addition to the spectroscopy, we have photometry obtained with HAWKI-I and NACO from Schödel et al. (2010) and Nogueras-Lara et al. (in preparation) in the $J$ (HAWK-I), $H$ (HAWKI-I and NACO), and $K_{S}$ (HAWKI-I and NACO) bands. Since the brightest stars can be saturated in the HAWKI-I and NACO images, we complemented the photometry with the SIRIUS catalogue (Nishiyama et al. 2006) for six late-type stars. For two further bright late-type stars with neither HAWK-I, NACO nor SIRIUS photometry due to saturation, we used Two Micron All Sky Survey (2MASS; Skrutskie et al. 2006) photometry.

We corrected the photometry for dust extinction using the extinction map and extinction law of Schödel et al. (2010), which was derived with NACO data. This extinction map covers about 70 per cent of our stars. For stars outside the field of view of the Schödel et al. (2010) extinction map, we used the extinction map of Nogueras-Lara et al. (in preparation) derived from HAWK-I data. It has lower spatial resolution, but covers the entire field of view of the KMOS data.

The $H-K_{S}$ colour can be used to identify foreground stars. The intrinsic $H-K_{S}$ colour ranges from about -0.13 to $+0.38 \mathrm{mag}$ (Do et al. 2013; Schödel et al. 2014b). We assumed that stars with significantly bluer extinction-corrected $\left(H-K_{S}\right)_{0}$ colour are overcorrected foreground stars. We classified a star as foreground star when $\left(H-K_{S}\right)_{0}<-0.5 \mathrm{mag}$. Including zero-point uncertainties, the uncertainty of the $H$ - and $K_{S}$-band magnitudes in our photometry is less than 10 per cent, so $\left(H-K_{S}\right)_{0}<-0.5 \mathrm{mag}$ corresponds to a $3 \sigma$ exclusion criterion. We excluded 62 stars with this criterion. For 11 stars, we do not have photometry in both bands. We excluded these 11 stars in our later analysis, since they might be foreground stars. In our selected data set, the extinction corrected $K_{S}$-band magnitudes range from 4.91 to $13.56 \mathrm{mag}$, with a median of $10.49 \mathrm{mag}$. For a colour-magnitude diagram of the data set, we refer to Feldmeier-Krause et al. (2015).

\subsection{Spectral indices}

Spectral indices in the $K$ band are correlated with the spectral type of a star, and can be used for a rough spectral classification and effective temperature estimate (e.g. Ivanov et al. 2004; Silva et al. 2008). The most prominent spectral features of cool stars are the $\mathrm{CO}$ absorption lines $(\lambda \geqslant 22935)$ and the Na I doublet at 22062 and 22090 . In this section, we calibrate the $\mathrm{CO}$ equivalent width $\left(E W_{\mathrm{CO}}\right)$ and the $\mathrm{Na}$ equivalent width $\left(E W_{\mathrm{Na}}\right)$ on a spectral library and present the measurements on our data set. The $E W_{\mathrm{CO}}$ and $E W_{\mathrm{Na}}$ measurements confirm our classification as late-type stars quantitatively. Further, we obtain some constraints on the effective temperature $T_{\text {eff }}$ and surface gravity $\log (g)$ that are useful as priors for the full-spectral fitting.

\subsubsection{Calibrating spectral indices with a spectral library}

We measured the spectral indices $E W_{\mathrm{CO}}$ and $E W_{\mathrm{Na}}$ using different spectral libraries with known spectral types for calibration. The spectral libraries are listed in Table 1. We used the spectral index definitions of Frogel et al. (2001). All spectra were degraded to the same spectral resolution of the KMOS data before we computed the indices. The spectral resolution of the KMOS detector varies for the different IFUs (see Section 2.2). We tested the effect of the spectral resolution by degrading the spectral library to $R=3000$, and found no systematic difference in the result compared to the spectral indices obtained with $R=4350$. The difference is less than 1 per cent for all indices. This test shows that equivalent width measurements are robust under moderate spectral resolution variations. Since the results with $R=3000$ are consistent with the results at KMOS resolution, we included spectra from Wallace \& Hinkle (1997, $R=3000)^{1}$ to obtain a larger sample. We also tested the influence of the continuum shape on index measurements by

1 We excluded stars that are also in the sample of Wallace \& Hinkle (1996), and the star HR8530/HD212320, which was classified as M6III star by Wallace \& Hinkle (1997), but was listed as G6III star by McWilliam (1990). 
reddening the library spectra with the mean extinction in our field of view, $A_{K_{S}}=2.7 \mathrm{mag}$. For the $\mathrm{CO}$ and $\mathrm{Na}$ equivalent width measurements the effect is less than 1 per cent, though it is about 7.3 per cent when we instead compute the $D_{\mathrm{CO}}$ index as defined by Mármol-Queraltó et al. (2008).

Giant stars with luminosity class II-IV have a mean value for $E W_{\mathrm{CO}}$ of approximately 13 , and the maximum value is about 25 . We expect that the majority of the stars in our data set are giants. However, we also computed $E W_{\mathrm{CO}}$ for the supergiant and dwarf stars in our spectral library. Supergiants have larger values of $E W_{\mathrm{CO}}$, with a mean at 20 , and values up to 33 . Since no giant star has a larger value of $E W_{\mathrm{CO}}$ than 25 , we can assume that stars in our data set with $E W_{\mathrm{CO}}>25$ are either M-type supergiants, or have a higher metallicity, as $\mathrm{CO}$ absorption strength also increases with metallicity (e.g. Houdashelt et al. 1992 for $[\mathrm{Fe} / \mathrm{H}]<0 \mathrm{dex})$. The mean value of $E W_{\mathrm{Na}}$ is about 2 for giant stars and about 3 for supergiants. We further found that all stars with a detectable CO line, from dwarfs over giants to supergiants, have effective temperatures $T_{\text {eff }} \lesssim 6000 \mathrm{~K}$. Since all the stars in our data set have $\mathrm{CO}$ lines, their temperatures are probably $\lesssim 6000 \mathrm{~K}$. We derived a new $T_{\text {eff }}-E W_{\mathrm{CO}}$ relation for giant stars. The results are presented in Appendix A.

\subsubsection{Late-type star classification}

To verify our classification as late-type stars, we measured spectral indices on our data set. We computed the equivalent widths of the first $\mathrm{CO}$ band head and the Na I doublet as defined by Frogel et al. (2001). Before computing the spectral indices, we measured the radial velocities of the stellar spectra and shifted the spectra to rest wavelength. To obtain the velocities, we used the IDL routine $p P X F$ (Cappellari \& Emsellem 2004) as in Feldmeier et al. (2014), with the high resolution spectra of Wallace \& Hinkle (1996) as templates. The wavelength range of the fit was from 21500 to 23900 .

The late-type stars have mean values of $E W_{\mathrm{CO}, \mathrm{LT}}=18.30$ and $E W_{\mathrm{Na}, \mathrm{LT}}=4.60$. The stars we classified as early-type $\mathrm{O} / \mathrm{B}$ stars have smaller equivalent widths, with a mean value of $E W_{\mathrm{CO}, \mathrm{O} / \mathrm{B}}=-0.76$ and $E W_{\mathrm{Na}, \mathrm{O} / \mathrm{B}}=0.47$ (see also Feldmeier-Krause et al. 2015). There is a clear distinction between the distribution of the $E W_{\mathrm{CO}}$ of the early- and late-type stars, which confirms our visual classification. Our data set contains 66 stars with $E W_{\mathrm{CO}}>25$, which might suggest that these stars are supergiants. Among those stars, 47 are rather bright $\left(K_{S, 0} \leqslant 10 \mathrm{mag}\right)$ giving further support to those objects being supergiants at the Galactic Centre. The CO strength in Galactic Centre supergiants is normal in comparison to disc stars (Blum et al. 1996).

The Na I doublet lines are rather strong for most stars in the data set, with a mean value of $E W_{\mathrm{Na}}=4.60$, while the giant stars in the spectral library have $E W_{\mathrm{Na}} \leqslant 4.5$. Blum et al. (1996) showed that stars in the Galactic Centre have higher $\mathrm{NaI}$ and $\mathrm{Ca}$ I line strengths compared to disc stars with similar $\mathrm{CO}$ strengths. Also the high-resolution spectra studied by Cunha et al. (2007) and Ryde \& Schultheis (2015) have high $[\mathrm{Ca} / \mathrm{Fe}]$ abundances. We show three example spectra in Fig. 1. The $\mathrm{NaI}$ and $\mathrm{Ca}$ I line regions are marked as grey shaded areas. $\mathrm{Na}$ is produced in massive stars in SN II (Kobayashi et al. 2006) and in intermediate-mass AGB stars (Karakas 2010). In contrast to $\mathrm{Na}, \mathrm{Ca}$ is an $\alpha$ - element. The Na I lines are blended with other elements, e.g. $\mathrm{Sc}, \mathrm{Si}, \mathrm{Fe}, \mathrm{V}$, and CN lines (Wallace \& Hinkle 1996). One or several of these elements might be enhanced and produce the large Na I equivalent width.

\section{FULL-SPECTRAL FITTING}

We fitted the spectra of our KMOS data set using the StARKIT code (Kerzendorf \& Do 2015) also used by Do et al. (2015). This code interpolates on a grid of synthetic spectra and utilizes the Bayesian sampler MultiNest (Feroz et al. 2009; Buchner et al. 2014). In the following, we give a short outline of our assumptions and used parameters. For details on the Bayesian sampling procedure we refer to Do et al. (2015).

\subsection{Fitting method and assumptions}

We fitted the effective temperature $T_{\text {eff }}$, metallicity $[M / H]$, surface gravity $\log (g)$, and radial velocity $v_{z}$. $[\alpha / F e]$ was fixed to zero and stellar rotation was ignored. Most of the stars in our data set are red giants, which have too low rotational velocities to be resolved with our data $\left(<10 \mathrm{~km} \mathrm{~s}^{-1}\right.$; Oke \& Greenstein 1954; Gray 1989). Our spectral grid consists of the model spectra from the PHOENIX spectral library (Husser et al. 2013), with stellar parameters in the range $T_{\text {eff }}=[2300 \mathrm{~K} ; 12000$ $\mathrm{K}]$ with a step size of $\Delta T_{\mathrm{eff}}=100 \mathrm{~K},[M / H]=[-1.5 \mathrm{dex}$, $+1.0 \mathrm{dex}], \quad \Delta[M / H]=0.5 \mathrm{dex}, \quad \log (g)=[0.0 \mathrm{dex}, \quad 6.0 \mathrm{dex}]$, $\Delta \log (g)=0.5$ dex. The synthetic spectra have a resolution of $R=500000$. We convolved the spectra to the same spectral resolution as the KMOS spectra. We took the different spectral resolutions obtained on the 23 different IFUs into account (see Section 2.2). The prior of the metallicity $[M / H]$ was set uniform in the range $[-1.5 \mathrm{dex},+1.0 \mathrm{dex}]$. The surface gravity can be constrained by the $K_{S}$-band magnitude, as shown by Do et al. (2015). Stars brighter than $K_{S}=12 \mathrm{mag}$ have a lower surface gravity. Therefore, we set the uniform priors for $\log (g)$ in the range $2.0 \mathrm{dex}<\log (g)<4.5 \mathrm{dex}$ for $K_{S, 0} \geqslant 12 \mathrm{mag}$ and to $0.0 \mathrm{dex}<\log (g)<4.0 \mathrm{dex}$ for $K_{S, 0}<12 \mathrm{mag}$. In addition, we chose $0.0 \operatorname{dex}<\log (g)<2.0$ dex for all stars with $K_{S, 0} \leqslant 10 \mathrm{mag}$ and $E W_{\mathrm{CO}}>25$, and $0.0 \operatorname{dex}<\log (g)$ $<4.0$ dex for all stars with $K_{S, 0}>10 \mathrm{mag}$ and $E W_{\mathrm{CO}}>25$, as these stars are potentially supergiants (see Section 2.4).

The continua of the spectra were not normalized or straightened. Still, we did not use the continuum shape to constrain the effective temperature, as interstellar dust and extinction also affect the continuum shape and might bias our results for the stellar parameters. We fitted the spectral continuum with a fifth degree polynomial function, to minimize the difference between the observed and model spectrum. The model spectra were multiplied with the polynomial to match the continuum shape of the data.

We fitted the spectra in the wavelength range $\lambda=[20900,22900]$, i.e., we excluded the molecular CO absorption lines from the stellar parameter fit. Do et al. (2015) showed that fitting the spectrum in the CO line region can introduce significant biases in $\log (g)$ and $[M / H]$. The reason is probably that molecular lines are not as reliable as atomic lines in synthetic spectra. Since the $\mathrm{Na}$ and $\mathrm{Ca}$ lines 


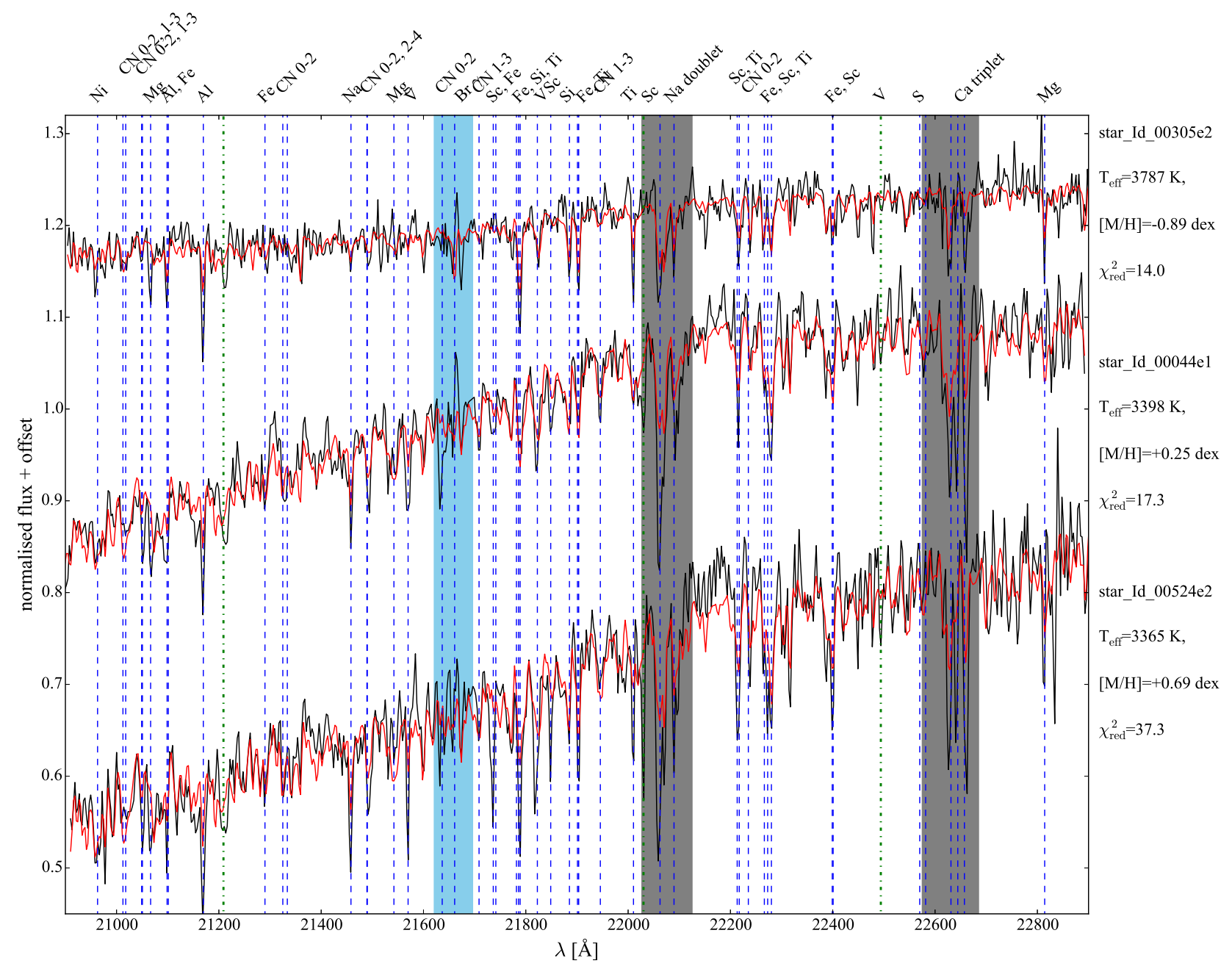

Figure 1. Spectra (black) of three different stars with metallicities $[M / H]=-0.89,+0.25$, and +0.69 dex. The red lines denote the best-fitting spectrum. The fits obtained similar values for $\log (g)$, between 0 and 0.34 dex, and $T_{\text {eff }}$, between 3360 and $3790 \mathrm{~K}$. The spectra are shifted to rest wavelength. Blue dashed lines denote known spectral lines, labelled on top of the plot, green dot-dashed lines spectral lines which appear in the observed spectra, but are not seen in the model spectra. Grey shaded areas were excluded from the fit, the light blue shaded area was excluded for spectra with strong contamination of the Brackett- $\gamma$ gas emission line.

of Galactic Centre stars are strong compared to normal disc stars (see Section 2.4.2 and Blum et al. 1996), we excluded these lines from the stellar parameter fit. In particular, we excluded the wavelength regions $\lambda=[22027,22125]$ and $\lambda=[22575,22685]$. However, we included the Na I and Ca I lines in the fit of the radial velocity $v_{z}$. For spectra with strong Brackett- $\gamma$ gas contamination emitted by the minispiral (for a Brackett- $\gamma$ flux map extracted from our data see Feldmeier-Krause et al. 2015), we also excluded the region $\lambda=[21621,21696]$ from the fit. Fig. 1 shows three spectra in the fitted wavelength region. The excluded Na I and Ca I line regions are shaded in grey. The Bracket- $\gamma$ line region, which was excluded for spectra with strong gas contamination, is shaded in light blue. Even though we excluded these regions, there are several other absorption lines in the spectra, some of them are marked with blue vertical dashed lines and labelled on top of the plot. The list of absorption lines we labelled is not complete. There are more lines visible in the spectra, and these lines affect the result of the fit.

\subsection{Error estimation}

\subsubsection{Statistical uncertainties}

We fitted the spectra of 907 stars. We selected spectra with high fitted signal to noise $\left[\mathrm{S} / \mathrm{N}_{\text {fit }}=\right.$ median $($ spectrum $) / \sigma($ residual $\quad$ spectrum $)>15$, where the residual spectrum equals observed spectrum minus best-fitting spectrum], and with low $1 \sigma$ uncertainties $\left(\sigma_{T_{\text {eff }}}<250 \mathrm{~K}, \sigma_{[M / H]}<0.25 \mathrm{dex}, \sigma_{\log (g)}<1 \mathrm{dex}\right)$. For stars with several exposures, we took the mean of the stellar parameters obtained by the individual fits as our measurement. In many cases, the standard deviation of the results from fitting the single exposures of the same star is larger than the $1 \sigma$ fit uncertainties. For these stars, we used the standard deviations as statistical uncertainties. We estimated the uncertainties of 230 stars with only one exposure from the median uncertainties of the other fits. The mean statistical uncertainties, either directly 


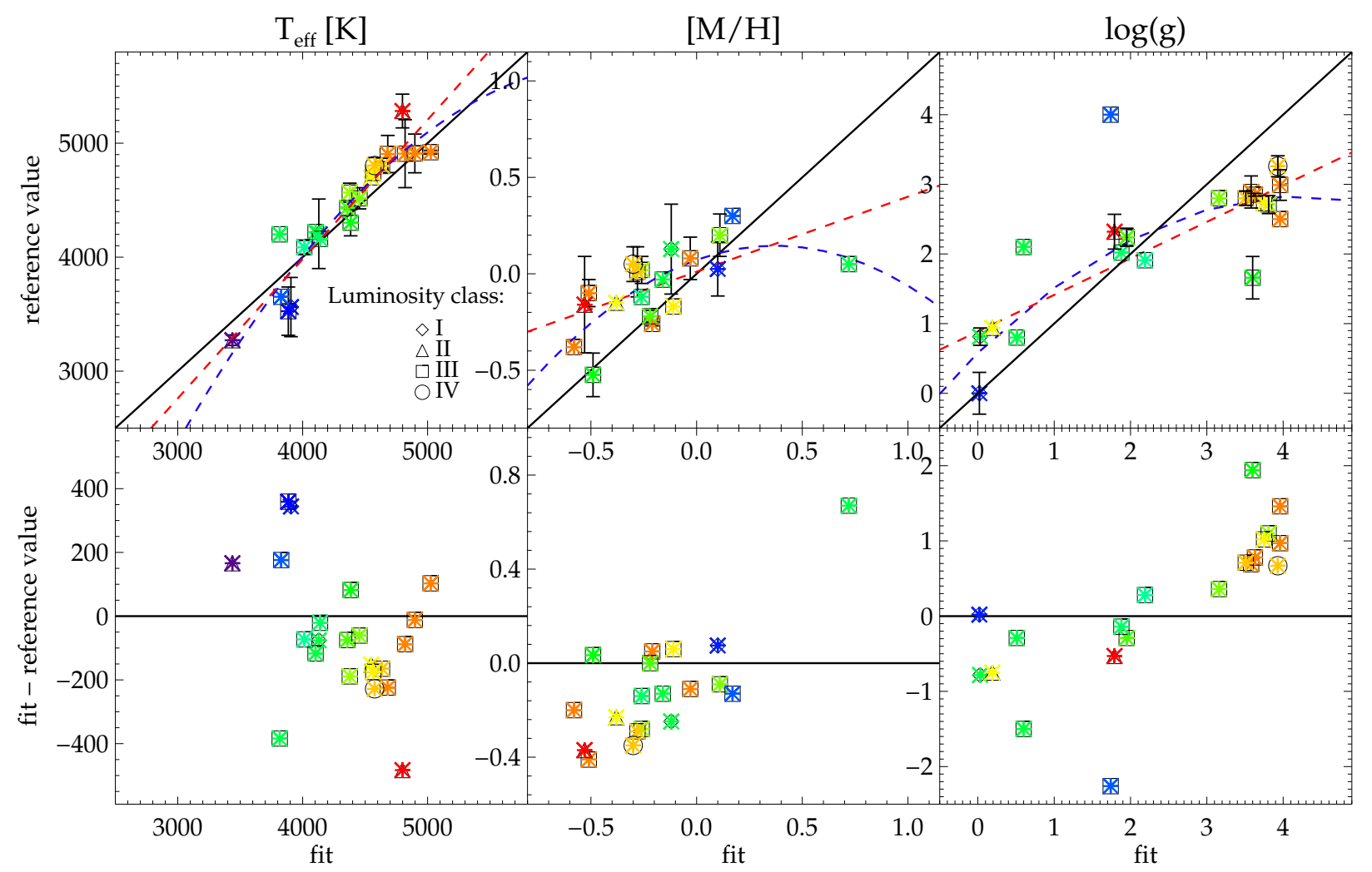

Figure 2. Comparison of the best-fitting stellar parameters with the reference values of stars from the spectral library. Left-hand panel: $T_{\text {eff }}$ in K for 22 stars; middle panel: $[M / H]$ in dex for 19 stars; right-hand panel: $\log (g)$ in dex for 20 stars. Upper panel: fit result plotted against the reference value, lower panel: fit result plotted against residual (fit result - reference value). The different symbols denote different luminosity classes, colours mark the same star. The black line is the one-to-one line, the red dashed line is a linear fit to the data, the blue dashed line a quadratic fit.

from the fits or the standard deviations, are $\sigma_{T_{\text {eff }}}=83 \mathrm{~K}$, $\sigma_{[M / H]}=0.11 \mathrm{dex}, \sigma_{\log (g)}=0.16 \mathrm{dex}$, and $\sigma_{v_{z}}=2.9 \mathrm{~km} \mathrm{~s}^{-1}$.

One of the reasons for different results of single exposures of the same star are so-called fringes or ripples (Neeser et al. 2016). Fringes are visible as wavy pattern in the continuum of the spectrum. Fringes can arise when the star is located at the edge of the IFU, or is spatially undersampled. Fortunately, stars that are close to the edge of the IFU have often more than two exposures. The standard deviation of the individual measurements gives a good estimation for the uncertainty of the parameter fit. In some cases, the standard deviation is very high. We conclude that the fit did not work properly for at least one of the exposures in such a case. We excluded stars with high statistical uncertainties.

\subsubsection{Systematic uncertainty of synthetic model spectra}

We determined systematic uncertainties by applying the fullspectral fitting routine on stars from a spectral library. We selected stars from Wallace \& Hinkle (1996) and Winge et al. (2009) for which previous measurements of the stellar parameters are listed in Soubiran et al. (2010). All the spectra were convolved to the KMOS spectral resolution. The GNIRS stars from Winge et al. (2009) cover a smaller wavelength range than our fitting interval, so we fitted those stars from $\lambda=[21800 ; 22900]$. We put constraints on $\log (g)$ based on the luminosity class (see also
Do et al. 2015). Giant stars with luminosity class III were constrained to the interval $\log (g)=[0.5 \mathrm{dex}, 4 \mathrm{dex}]$, supergiants and bright giants (luminosity class I and II) had $\log (g)=[0 \mathrm{dex}, 2 \mathrm{dex}]$. Subgiants (luminosity class IV) were constrained to $\log (g)=[2 \mathrm{dex}, 5.5 \mathrm{dex}]$. We did not include main-sequence stars (luminosity class $\mathrm{V}$ ), since we do not expect any dwarf stars in our data set except foreground stars. Even the faintest star in our data set with $K_{S}=16.9 \mathrm{mag}$ is too bright to be a cool main-sequence star in the Galactic Centre, whereas hot main-sequence stars (O/B/A-type) are not late-type stars and therefore excluded from the data set. In total, we fit 22 reference spectra with known $T_{\text {eff }}$, for 19 reference spectra we also know $[M / H]$, and for 20 stars $\log (g)$. We fitted each spectrum twice, we computed the standard deviation of the residual spectrum after the first fit and used it as uniform noise spectrum in the second fit. We show a comparison of the fitted results to the reference results in Fig. 2. The values of $T_{\text {eff }}$ are in good agreement, the mean and standard deviation of the fit residuals are $\left\langle\Delta T_{\text {eff }}\right\rangle=-58 \mathrm{~K}, \sigma_{\Delta T_{\text {eff }}}=205 \mathrm{~K},\langle\Delta[M / H]\rangle=-0.1 \mathrm{dex}$, $\sigma_{\Delta[M / H]}=0.24 \mathrm{dex},\langle\Delta \log (g)\rangle=0.2 \mathrm{dex}, \sigma_{\Delta \log (g)}=1.0 \mathrm{dex}$. To some extent, the scatter and offsets are caused by the different methods and assumptions used by the different studies that measured the reference stellar parameters. But as argued by Do et al. (2015), offsets are also due to systematics in the model spectra. We tested if the systematic uncertainties change as a function of $\mathrm{S} / \mathrm{N}$ by adding noise to the 


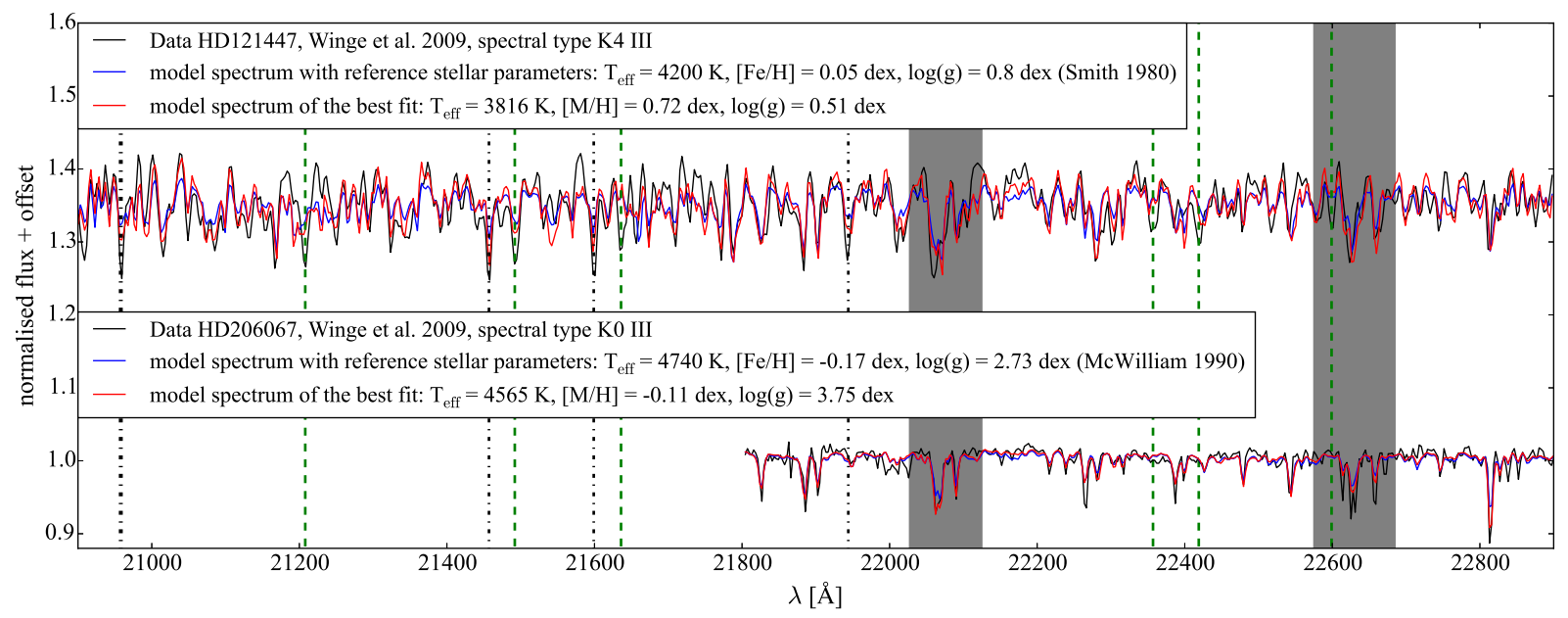

Figure 3. Top: NIFS spectrum of HD121447 (Winge et al. 2009, black), a PHOENIX (Husser et al. 2013) model spectrum with the reference values found by Smith $\left(1984\right.$, blue), and a model spectrum with the best-fitting results we obtained $\left(\right.$ red, $\left.\mathrm{S} / \mathrm{N}_{\mathrm{fit}}=44\right)$. Vertical dashed green lines denote spectral lines that are weaker in the model spectra, black dot-dashed lines denote spectral lines that are better recovered by a spectrum with higher metallicity. Bottom: GNIRS spectrum of HD206067 (Winge et al. 2009, black), our results (red, $\left.\mathrm{S} / \mathrm{N}_{\mathrm{fit}}=112\right)$ are in good agreement with the reference values found by McWilliam (1990, blue). Grey contours mark regions that were excluded from the fit.

reference spectra, but found consistent results for lower $\mathrm{S} / \mathrm{N}$. We added the standard deviations in quadrature to our statistical uncertainties to account for the systematics of the model spectra.

The systematic uncertainty values we obtained are lower than the systematic uncertainties found by Do et al. (2015). The reason for this is twofold. Do et al. (2015) used the MARCS grid (Gustafsson et al. 2008) and not the PHOENIX grid (Husser et al. 2013), and they used the IRTF SPEX library (Rayner et al. 2009) as reference stars. The IRTF stellar library has a spectral resolution of $R \approx 2000$. The MARCS grid was computed with different stellar abundances and atomic line lists than the PHOENIX synthetic spectra. We tested the MARCS grid for our reference stars and found $\left\langle\Delta T_{\text {eff }}\right\rangle=-38 \mathrm{~K}$, $\sigma_{\Delta T_{\text {eff }}}=268 \mathrm{~K},\langle\Delta[M / H]\rangle=-0.33 \mathrm{dex}, \sigma_{\Delta[M / H]}=0.25 \mathrm{dex}$, $\langle\Delta \log (g)\rangle=1.2 \mathrm{dex}, \sigma_{\Delta} \log (g)=0.7 \mathrm{dex}$. There appears to be a systematic offset to higher values of $\log (g)$ and lower values of $[M / H]$ compared to the reference values. Hence, we decided to use the PHOENIX synthetic spectra. However, there are also some systematic offsets when we use the PHOENIX grid. The effective temperature tends to be overestimated at $T_{\text {eff }} \lesssim 4000 \mathrm{~K}$ (by up to $360 \mathrm{~K}$, see lower left panel of Fig. 2), but rather underestimated at $T_{\text {eff }} \gtrsim 4500 \mathrm{~K}$ (by up to $480 \mathrm{~K}$ ). On the other hand, the results for $\log (g)$ are rather too high at $\log (g) \gtrsim 2.5$ dex, but too low at $\log (g) \lesssim 1$ dex. The metallicities of most spectra are in good agreement with the reference metallicities. We show two reference star fits in Fig. 3. We plotted the GNIRS spectrum of HD206067 (bottom, black). For this star, our results (red) are in good agreement with the reference stellar parameters (blue). There is one outlier for the metallicity results. The fit of the NIFS spectrum of the K4 III star HD121447 indicated a very high metallicity $([M / H] \gtrsim+0.72 \pm 0.05 \mathrm{dex})$, though the reference iron abundance is roughly solar $([\mathrm{Fe} / \mathrm{H}]=+0.05 \mathrm{dex}$; Smith 1984). The observed spectrum from Winge et al. (2009), the best- fitting model spectrum, and a model spectrum with the reference stellar parameters are shown in Fig. 3 (top). By eye, one can hardly decide which model spectrum fits the data better, however, some lines (marked with black dot-dashed vertical lines) are recovered better by our best fit than by the model with the reference values. There are some lines in the observed spectrum that are missing or only weak in the model spectra. Some of these lines are marked as green dashed vertical lines in Fig. 3. We conclude that fits, which obtain high metallicities $[M / H] \gtrsim+0.5$ dex, must be considered suspect. We note that we did not test our method on stars with high metallicities beyond $[M / H]=+0.3$ dex. As can be seen from Fig. 2, the fitted results in $T_{\text {eff }}$ appear to be very robust. A large discrepancy in the fitted metallicity or surface gravity still produces good results in $T_{\text {eff }}$.

\subsubsection{Total uncertainties}

We took different uncertainties into account and added them in quadrature. These uncertainties are (1) the statistical uncertainties, either the $1 \sigma$ fitting uncertainties or the standard deviation of several exposures of the same star and (2) systematic uncertainties of the synthetic model spectra. The systematic uncertainties are dominating the total uncertainty for most of the stars. The mean total uncertainties of our sample of stars are $\sigma_{T_{\text {eff }}}=230 \mathrm{~K}, \sigma_{[M / H]}=0.27 \mathrm{dex}$, $\sigma_{\log (g)}=1.04 \mathrm{dex}$ and $\sigma_{v_{z}}=2.9 \mathrm{~km} \mathrm{~s}^{-1}$. The mean statistical and total uncertainties are plotted as error bars in Fig. 4.

\section{RESULTS}

Our data set contains 982 stars in the central 64.9 arcsec $\times 43.3$ arcsec of the Milky Way, for which we fitted stellar parameters. We excluded several stars due to low signal-tonoise ratio or large uncertainties (Section 3.2.1). Stars with 

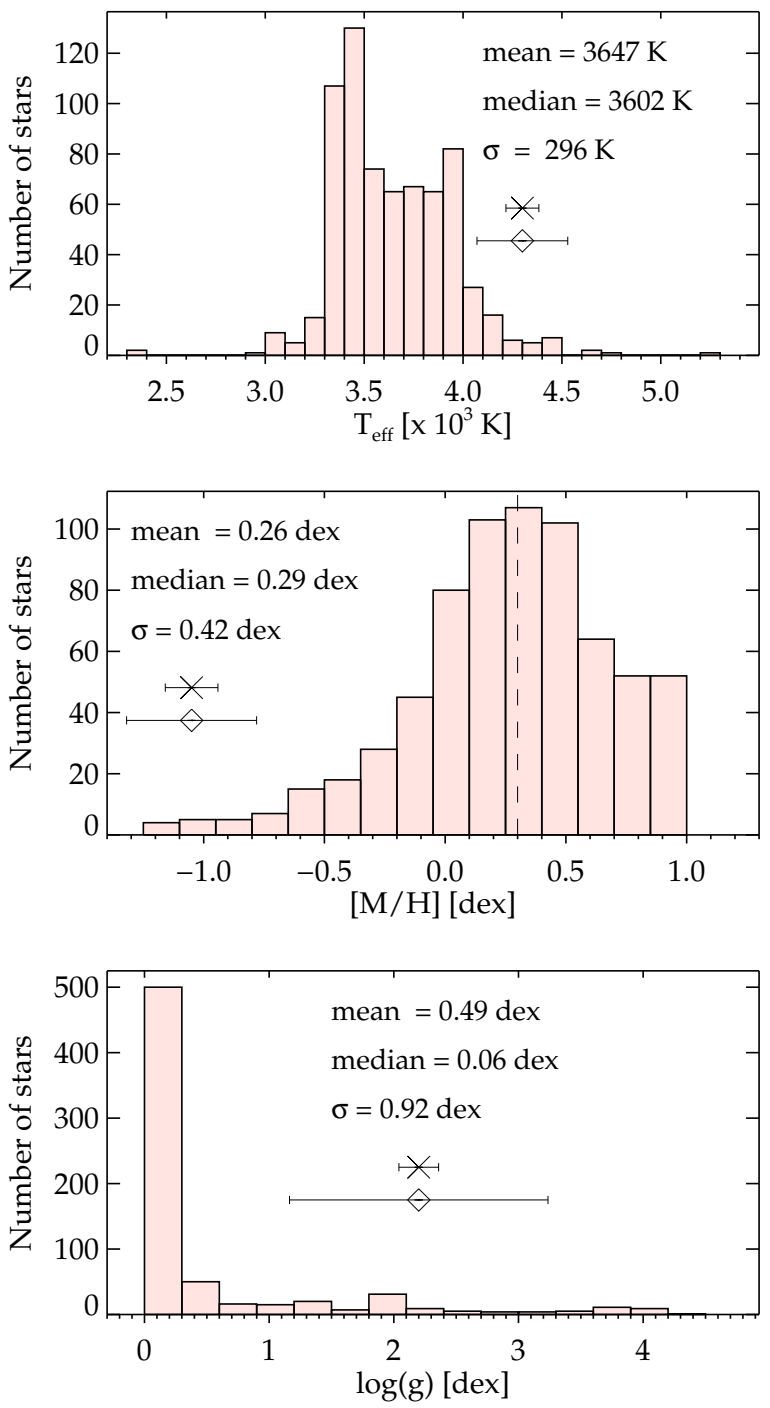

Figure 4. Histograms of the stellar parameters for 687 stars with $\left(H-K_{S}\right)_{0}=[-0.5 \mathrm{mag} ; 0.8 \mathrm{mag}]$, from top to bottom: effective temperature $T_{\text {eff }}$, metallicity $[M / H]$, surface gravity $\log (g)$. The error bars denote the mean statistical uncertainty (cross symbol), and mean total uncertainty (diamond symbol) of the measurements.

a rather blue colour, or no colour information, may be foreground stars, and not belong to the Milky Way nuclear star cluster. We excluded those 73 stars, which leaves 705 stars. A further 18 stars have rather red colours, they are probably locally embedded in dust, but might also be background stars. Fig. 4 illustrates the distribution of the stellar parameter results for 687 stars, which are probably cluster members, with extinction corrected colour $\left(H-K_{S}\right)_{0}=[-0.5 \mathrm{mag}, 0.8 \mathrm{mag}]$. The mean statistical and total uncertainties for the stellar parameter measurements are shown as error bars.

\subsection{Effective temperatures}

As expected, the late-type stars are mostly cool, with a mean temperature $\left\langle T_{\text {eff }}\right\rangle \approx 3650 \mathrm{~K}$ (upper panel of Fig. 4).
This means the stars are mostly late K-type stars or Mtype stars (Cox 2000). As shown in Section 3.2.2, we rather overestimate low temperatures and underestimate high temperatures. This indicates that the distribution of $T_{\text {eff }}$ might be too narrow. To estimate this effect, we made a linear fit to the $T_{\text {eff }}$ measurements of reference stars (red dashed line in upper left panel of Fig. 2). We shifted our $T_{\text {eff }}$ measurements using this relation, which broadens the $T_{\text {eff }}$ distribution by $77 \mathrm{~K}$. Only one star (Id 2164) has a temperature higher than $5000 \mathrm{~K}$, in agreement with a G-type star. Also the CO equivalent width is rather low (4.32), which confirms the higher effective temperature.

\subsection{Metallicities}

The mean metallicity is $\langle[M / H]\rangle=+0.26$ dex, this means that most stars are metal-rich. The standard deviation of $[M / H]$ is $0.42 \mathrm{dex}$. The metallicity distribution is shown on the middle panel of Fig. 4 for 687 likely cluster member stars. We found several metal-poor stars. Our data contain 156 stars with $[M / H]<0$ dex, from which 37 stars have $[M / H] \leqslant-0.5$ dex and 8 stars even $[M / H] \leqslant-1.0$ dex. One of the metal-poor stars with $[M / H] \leqslant-0.5$ dex has a rather red colour $\left[\left(H-K_{S}\right)_{0}=0.84 \mathrm{mag}\right]$, it might either be locally embedded or a background star. Our data set contains $\left(5.2_{-3.1}^{+6.0}\right)$ per cent low-metallicity stars (i.e. $[M / H] \leqslant-0.5 \mathrm{dex})$, and $\left(22.8_{-12.6}^{+24.5}\right)$ per cent subsolar metallicity stars $([M / H] \leqslant 0.0$ dex $)$.

Several stars are close to the boundary metallicity of the grid at $[M / H]=+1$ dex. This would mean that these stars are super-metal-rich. However, there is probably a problem with the fit or the spectral grid. We only tested the metallicity measurement on reference stars with $[M / H] \lesssim$ +0.3 dex. We indicated this metallicity as a vertical dashed line in the middle panel of Fig. 4. We cannot say whether our method works for stars with a higher metallicity. Further, we showed in Section 3.2.2 that the metallicity of a star with $[M / H]>0$ dex was overestimated by nearly 0.7 dex. We conclude that stars with a metallicity $[M / H] \gtrsim$ +0.3 dex are probably metal-rich $([M / H] \gtrsim 0$ dex $)$, however, with our method and models at moderate spectral resolution we cannot determine the metallicity to a higher accuracy. Fig. 1 shows three spectra with different metallicities $[M / H]=-0.89,+0.25$ and +0.69 dex. Their effective temperatures and surface gravities are very similar. These spectra have signal-to-noise values of $\mathrm{S} / \mathrm{N}_{\text {fit }} \geqslant 30$. For all three spectra, the Na doublet and Ca triplet lines (marked as grey shaded area) are deeper than in the best-fitting spectra. For the metal-poor star, many of the other lines are fitted reasonably well, e.g. the Fe lines at 21 290, 21 782, 21 901, 22266 , or 22399 , which are blended with $\mathrm{Ti}, \mathrm{Si}$, or Sc. The lines at 21290,21782 , and 21901 are also fitted well for the spectrum with $[M / H]=+0.25$ dex. However, in particular the spectrum with the highest metallicity $[M / H]=+0.69 \mathrm{dex}$ has many lines that are deeper than the best-fitting model spectrum. This confirms that the stars with a best-fitting metallicity $[M / H]>+0.3$ dex are definitely very interesting targets to be followed-up with high-resolution spectroscopy. At this point, it would be premature to claim that these stars are indeed super-metal-rich. 


\subsection{Surface gravities}

The surface gravity is mostly low, in agreement with cool giant $[\log (g)<3.0 \mathrm{dex}]$ and supergiant $[\log (g)<1.5 \mathrm{dex}]$ stars (lower panel of Fig. 4). For most stars we obtained a value of $\log (g)$ close to zero, at the edge of the PHOENIX spectral grid. This suggests that the value of $\log (g)$ might be even negative, as for M-type supergiants. However, we showed in Section 3.2.2 that $\log (g)$ is rather underestimated at low values of $\log (g)$, and the systematic uncertainty is 1 dex. Therefore, we consider the results of $\log (g)$ as highly uncertain.

We fitted the surface gravity together with the other stellar parameters but treated it mostly as a nuisance parameter, rather than an actual measurement. We did this to avoid any biases that may be introduced by fixing $\log (g)$ to a wrong value. As we showed in Section 3.2.2, fitting a discrepant value in $\log (g)$ does not directly translate into a wrong value for the effective temperature or metallicity. Some of the most discrepant results for $\log (g)$ give very good results for $T_{\text {eff }}$ and $[M / H]$.

\subsection{Radial velocities}

We fitted the radial velocities together with the stellar parameters. They range from -268.8 to $+313.6 \mathrm{~km} \mathrm{~s}^{-1}$. The mean velocity of 705 stars with $\left(H-K_{S}\right)_{0}>-0.5 \mathrm{mag}$ is $\left\langle v_{z}\right\rangle=+7.6 \pm 3.6 \mathrm{~km} \mathrm{~s}^{-1}$. The disagreement from zero is probably due to the asymmetric spatial distribution of stars in our data set, with 410 stars in the Galactic East, and 295 stars in the Galactic West. The velocity dispersion $\left\langle\sigma_{z}\right\rangle=96.2 \pm 2.6 \mathrm{~km} \mathrm{~s}^{-1}$. We computed the mean velocity and velocity dispersion with the maximum likelihood approach (Pryor \& Meylan 1993), which takes the individual velocity uncertainties into account.

We also considered the Galactic East and Galactic West separately. We obtained for the 410 stars in the Galactic East $\left\langle v_{z}\right\rangle=+24.6 \pm 4.8 \mathrm{~km} \mathrm{~s}^{-1},\left\langle\sigma_{z}\right\rangle=96.4 \pm 3.4 \mathrm{~km} \mathrm{~s}^{-1}$, and $\left\langle v_{z}\right\rangle=-16.2 \pm 5.3 \mathrm{~km} \mathrm{~s}^{-1},\left\langle\sigma_{z}\right\rangle=90.7 \pm 3.8 \mathrm{~km} \mathrm{~s}^{-1}$ for the 295 stars in the Galactic West. The mean radial velocity confirms the rotation of the Milky Way nuclear star cluster by approximately $20 \mathrm{~km} \mathrm{~s}^{-1}$ within the central $1.2 \mathrm{pc}$ (30 arcsec), as found by McGinn et al. (1989) and also Feldmeier et al. (2014) from a different data set.

\subsection{Long-period variable stars}

Our data contain six stars with $T_{\text {eff }}<3000 \mathrm{~K}$, five of them have an effective temperature close to the edge of the PHOENIX grid, at $T_{\text {eff }}=2300 \mathrm{~K}$. We checked those stars in the SIMBAD data base (Wenger et al. 2000) and found that four of the stars were classified as long-period variables or Mira stars (Id 158, 221, 25349, 25389) by Reid et al. (2003) and Matsunaga et al. (2009). Such stars are mostly M-type giants or supergiants, with periods of few to several hundred days. However, long-period variables can also be carbon-rich (C-type stars), or zirconium-rich (S-type stars), and Mira stars can be oxygen-rich or carbon-rich (Cox 2000).

The six stars are rather bright, with observed magnitudes ranging from $K_{S}=9$ to $12.3 \mathrm{mag}$. This also indicates that the stars are supergiant and bright giant stars, however, we obtained $\log (g)$ values ranging from 1.5 to
5.6 dex. We conclude that the PHOENIX spectral grid is not suitable to obtain the surface gravity of C-type, S-type or oxygen-rich long-period variable stars. Many Mira stars suffer from rapid mass-loss. These stars are often embedded in dust, which causes reddening. Indeed, three of these six cool stars have rather red extinction-corrected colours $\left[\left(H-K_{S}\right)_{0}=0.93-2.12 \mathrm{mag}\right]$. This reddening makes it hard to classify the six cool stars as member or background stars. Further, all six stars have a steeply rising continuum (slope $\mathrm{m}=\Delta$ flux $/ \Delta \lambda=3.2-4.8$, see also Fig. 5), at least three times steeper than most other stars. This also indicates that the stars are embedded in dust, and our photometric extinction correction was possibly too low.

\subsection{Spatial distribution and kinematics of metal-poor stars}

We investigated if the metal-poor stars show any kind of peculiar spatial distribution. For this purpose, we counted the number of metal-poor stars $([M / H] \leqslant-0.5)$ in circular annuli around Sgr $A^{*}$. We chose the bins such that each bin contains at least five metal-poor stars. The fraction of metal-poor stars divided by the total number of late-type stars is shown in the upper panel of Fig. 6. The uncertainties come from the total uncertainty of $[M / H]$. We did not find a significant change of the fraction of metal-poor stars in the range of $0-1.4 \mathrm{pc}$ projected distance from Sgr A*. Further, we divided the data set in eight segments, and computed the fraction of metal-poor stars with respect to the total number of stars as a function of the position angle. The result is shown in Fig. 6 (lower panel). We found that the fraction of metal-poor stars is highest in the Galactic North East, and lower in the Galactic South. But again, the uncertainties are too high for this trend to be significant.

We show a completeness corrected surface number density profile of metal-poor stars (red triangles) and metal-rich stars $([M / H] \geqslant 0.0$ dex, green diamond symbols $)$ in Fig. 7 . We used the same method as in Feldmeier-Krause et al. (2015) to construct the profile, i.e. we corrected the number counts in different magnitude and radial bins for completeness, and counted the stars in rings around Sgr A*. The surface number density of early-type stars is denoted with blue square symbols. The decrease of the early-type star density to larger radii is steep, over more than two orders of magnitude. The density of stars with $[M / H] \geqslant 0.0$ dex and $K_{S}<14$ mag is nearly constant, and decreases only by a factor $\lesssim 3$. There is much scatter in the surface number density of metal-poor stars.

We also illustrate the spatial distribution of the 705 stars with $\left(H-K_{S}\right)_{0}>-0.5 \mathrm{mag}$ in Fig. 8. The upper panel is colour-coded with the metallicity $[M / H]$, and metalpoor stars are highlighted as square symbols. The middle panel is colour-coded with the effective temperature. Metal-poor stars are rather hotter than the other stars, with $\left\langle T_{\text {eff }}\right\rangle=3980 \mathrm{~K}$. Only one metal-poor star is a long-period variable star with a low temperature of $2300 \mathrm{~K}$. We already discussed in Section 4.5 that long-period variable stars can have an unusual chemical composition, and therefore the results are uncertain.

The radial velocity $v_{z}$ is illustrated in the lower panel of Fig. 8. The metal-poor stars also rotate around Sgr $A^{*}$, and their mean velocity and velocity dispersion 

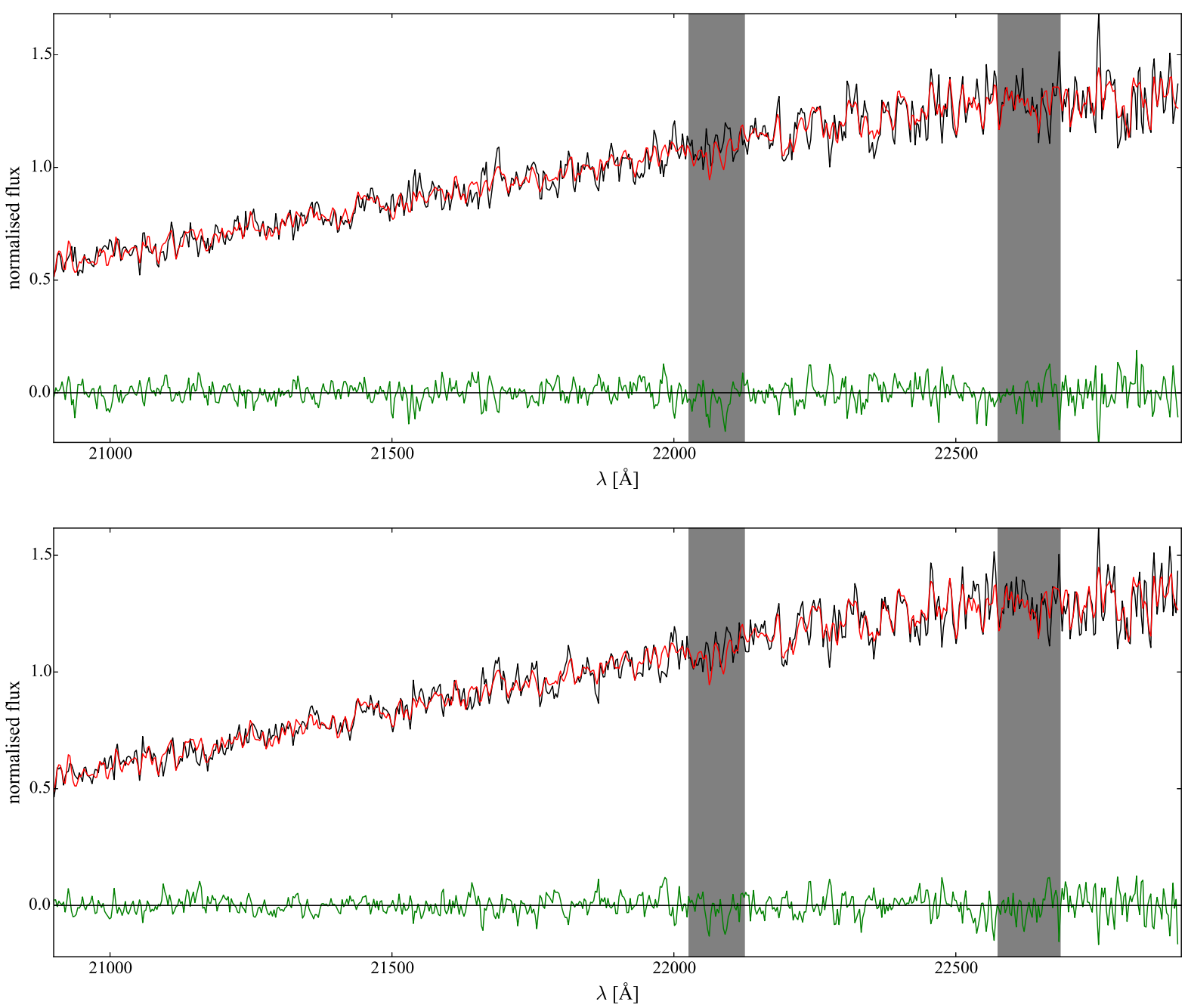

Figure 5. Spectra of the long-period variable star Id 649 in black. We fit the two exposures separately, the best fit (red) is obtained at $T_{\text {eff }}=2300 \mathrm{~K},[M / H]=+0.99 \mathrm{dex}, \log (g)=5 \mathrm{dex}$. Green circles denote the fit residuals. The flux of the spectrum increases towards longer wavelengths. The fits have $\chi_{\text {red }}^{2}=11.6$ and 14.2 , and $\mathrm{S} / \mathrm{N}_{\text {fit }}=20.6$ and 22.5 .

are $\left\langle v_{z}\right\rangle=+76.8 \mathrm{~km} \mathrm{~s}^{-1}, \sigma_{z}=101.9 \mathrm{~km} \mathrm{~s}^{-1}$ in the Galactic East, $\left\langle v_{z}\right\rangle=-15.2 \mathrm{~km} \mathrm{~s}^{-1}, \sigma_{z}=124.3 \mathrm{~km} \mathrm{~s}^{-1}$ in the Galactic West, and $\left\langle v_{z}\right\rangle=+47.0 \mathrm{~km} \mathrm{~s}^{-1}, \sigma_{z}=116.4 \mathrm{~km} \mathrm{~s}^{-1}$ in the entire field. The velocity dispersion is similar to the velocity dispersion of all stars $\left(\sigma_{z}=94.6 \pm 2.6 \mathrm{~km} \mathrm{~s}^{-1}\right.$, Section 4.4). We also matched the stars with the proper motion data of Schödel et al. (2009), and found 400 matches. The proper motions of the metal-poor stars are plotted as black arrows in Fig. 8. The average velocity dispersion over two dimensions for 21 metal-poor stars with proper motions $\left(\sigma_{2 d}=89.8 \mathrm{~km} \mathrm{~s}^{-1}\right)$ is in agreement with the velocity dispersion of the other stars $\left(\sigma_{2 d}=100.3 \pm 3.7 \mathrm{~km} \mathrm{~s}^{-1}\right)$. As for the radial velocities, the absolute two-dimensional velocity of the metal-poor stars $\left(\left\langle v_{2 d}\right\rangle=126.3 \mathrm{~km} \mathrm{~s}^{-1}\right)$ is in agreement with the two-dimensional velocity of the other 379 stars $\left(\left\langle v_{2 d}\right\rangle=127.5 \pm 3.3 \mathrm{~km} \mathrm{~s}^{-1}\right)$. Altogether the kinematics of the metal-poor stars are not significantly distinct from the kinematics of the other stars.

\subsection{Influence of radial velocity shift}

Depending on the radial velocity of a star, a slightly different wavelength region is fitted. In this section, we test if this has a strong effect. We fitted the spectra as observed in the fixed wavelength interval $\lambda=[20900,22900]$. But since the stars have velocities in the range of $v_{z}=\left[-269 \mathrm{~km} \mathrm{~s}^{-1}\right.$; $314 \mathrm{~km} \mathrm{~s}^{-1}$ ], some spectra are shifted with respect to the rest wavelength by up to 22 . This means we fit the stars in different rest wavelength intervals, and some lines at the edge of $\lambda=[20900,22900]$ may be part of the fit or not, depending on the stellar radial velocity. To test the magnitude of this effect, we shifted 50 spectra to the rest wavelength, and fitted the spectra at slightly shifted wavelength regions, at $\lambda=[20900,22900], \lambda=[20920,22920]$, and $\lambda=[20880$, $22880]$. The offset of \pm 20 corresponds to $\pm 270 \mathrm{~km} \mathrm{~s}^{-1}$. For these 50 spectra we found that the median of the stellar parameter change is $\Delta T_{\text {eff }}=3 \mathrm{~K}, \Delta[M / H]=+0.01 \mathrm{dex}$, $\Delta \log (g)=+0.01$ dex, $\Delta v_{z}=0.3 \mathrm{~km} \mathrm{~s}^{-1}$ with standard deviations of $\sigma_{\Delta T_{\text {eff }}}=44 \mathrm{~K}, \sigma_{\Delta[M / H]}=0.1 \mathrm{dex}, \sigma_{\Delta \log (g)}=0.6 \mathrm{dex}$, and $\sigma_{\Delta v_{z}}=0.7 \mathrm{~km} \mathrm{~s}^{-1}$. This means that a shift in the radial velocity does not bias the results of the stellar parameters, 

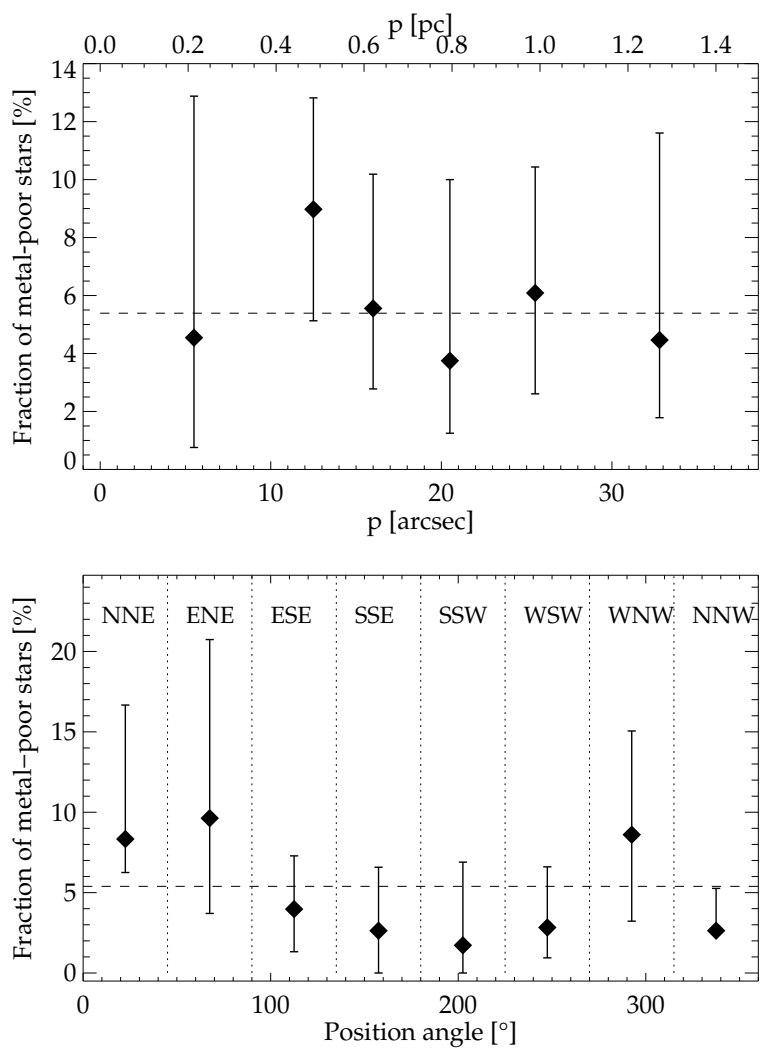

Figure 6. Fraction of metal-poor stars $([M / H] \leqslant-0.5 \mathrm{dex})$ as a function of projected distance $p$ from Sgr A* (upper panel), and as function of the position angle (Galactic East of North) centred on Sgr A* (lower panel).

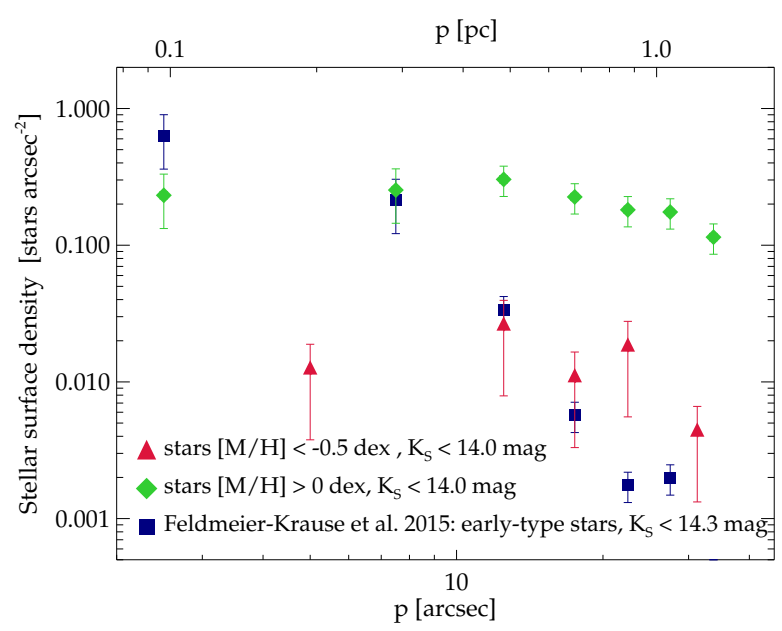

Figure 7. Completeness corrected stellar surface number density profile for stars brighter than $K_{S}=14.0 \mathrm{mag}$. Red triangles denote metal-poor stars $([M / H] \leqslant-0.5$ dex $)$, green diamond symbols denote supersolar metallicity stars $([M / H] \geqslant 0.0 \mathrm{dex})$. Blue square symbols illustrate the distribution of early-type stars.
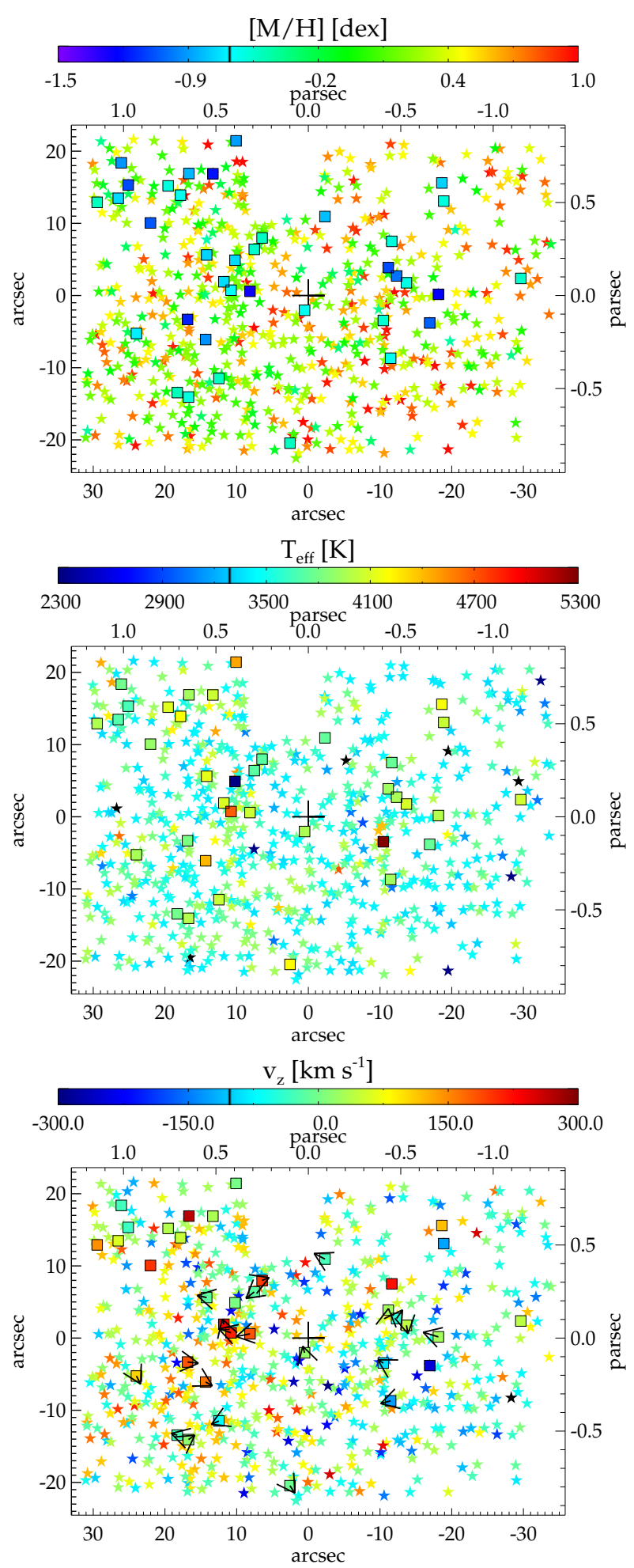

Figure 8. Spatial distribution of 705 stars with $(H-$ $\left.K_{S}\right)_{0}>-0.5 \mathrm{mag}$ in offset coordinates from the central supermassive black hole, Sgr A*, illustrated as black cross. Galactic North is up. The colours denote the metallicity $[M / H]$ (upper panel), effective temperature $T_{\text {eff }}$ (middle panel) and radial velocity $v_{z}$ (lower panel). The square symbols highlight metal-poor stars with $[M / H] \leqslant-0.5$ dex. Black arrows denote proper motions of metal-poor stars. 
though it can change the results. The difference is usually less than the uncertainties.

\subsection{Influence of spectral resolution}

As we showed in Section 2.2, the spectral resolution depends on the used IFU. For the spectral fits, we took the different spectral resolutions into account. In this section, we test if a lower spectral resolution obtains consistent results with a higher spectral resolution, and how a mismatch of the data and model spectral resolution affects the results.

We used the spectral library and convolved the spectra to the minimum and maximum spectral resolution of the KMOS data $(R=3300$ and 4660$)$. We fitted the spectra with $R=3300$ using model spectra at $R=4660$, and the spectra with $R=4660$ using model spectra at $R=$ 3300 . The results of $T_{\text {eff }}, \log (g)$ and $v_{z}$ change only slightly, and there are no systematic offsets. Only the metallicity is affected. In particular, when fitting the data with models that have a higher spectral resolution, the metallicity is shifted by $\langle\Delta[M / H]\rangle=+0.1$ dex with $\sigma_{\Delta[M / H]}=0.06$ dex. On the other hand, fitting data using model spectra that have a lower spectral resolution, the metallicity is shifted by $\langle\Delta[M / H]\rangle=-0.09$ dex with $\sigma_{\Delta[M / H]}=0.05$ dex.

We repeated the fits using model spectra convolved to the correct respective spectral resolution. The mean differences and standard deviations at the different spectral resolutions are small compared to the uncertainties. We conclude that a variation of the spectral resolution does not bias our results when the correct spectral resolution is adopted for each spectrum.

\section{DISCUSSION}

In this section, we discuss our results of the stellar metallicities, and compare them with other metallicity measurements. We argue that the stars in our data set are likely members of the nuclear star cluster. From the stellar $K$ band magnitude, we infer the luminosity classes and conclude that the metal-poor stars are consistent with globular cluster stellar populations. We finally discuss implications for the formation of the Milky Way nuclear star cluster.

\subsection{Detection of stars with low metallicity $[M / H] \lesssim-0.5$ dex}

We measured the metallicities of 705 stars in the nearinfrared $K$ band with medium spectral resolution. We detected 37 stars with $[M / H] \lesssim-0.5 \mathrm{dex}$, this is 5.2 per cent of the stars in our sample. Their mean magnitude $K_{S, 0}=13.18 \mathrm{mag}$ is similar to the mean magnitude of the stars with $[M / H]>-0.5 \operatorname{dex}\left(K_{S, 0}=13.04 \mathrm{mag}\right)$. We could not calibrate the metallicity measurements with metalpoor library stars with $[M / H]<-0.5$ dex. Therefore, we could not test if we can differentiate between stars with $[M / H]=-0.5$ dex and $[M / H]=-1.0$ dex. But we showed in Section 3.2.2 that our method is able to differentiate metal-poor $([M / H]<-0.3 \mathrm{dex})$ from metal-rich stars $([M / H]>+0.0 \operatorname{dex})$.
Our metallicity results are in agreement with the results of Do et al. (2015), who measured the metallicities of 83 Galactic Centre stars. Do et al. (2015) used the same fitting procedure as this work, but their data have a higher spatial and spectral resolution $(R=5400)$. Further, they used the MARCS grid (Gustafsson et al. 2008). We matched their 83 stars with our data set. To obtain a match, the distance of two stars has to be less than 0.3 arcsec, and the difference in $K_{S}$-band magnitude not more than $0.4 \mathrm{mag}$. With these conditions, we have 29 stars in common. The temperatures and metallicities are in reasonable agreement, with $\left\langle\Delta T_{\text {eff }}\right\rangle=-2.6 \mathrm{~K}$, $\sigma_{\Delta T_{\text {eff }}}=148 \mathrm{~K},\langle\Delta[M / H]\rangle=-0.32 \operatorname{dex}, \sigma_{\Delta[M / H]}=0.35 \mathrm{dex}$. The values of $\log (g)$ have a larger disagreement than expected from fitting the spectral library in Section 3.2.2, with $\langle\Delta \log (g)\rangle=-2.6 \operatorname{dex}, \sigma_{\Delta \log (g)}=0.9$ dex. This is caused by the low surface gravities obtained with the PHOENIX grid, and the tendency of the MARCS grid to higher surface gravities (see fig. 2 of Do et al. 2015). The radial velocity is also offset by $\left\langle\Delta v_{z}\right\rangle=28.9 \mathrm{~km} \mathrm{~s}^{-1}, \sigma_{v_{z}}=7.5 \mathrm{~km} \mathrm{~s}^{-1}$, but this is simply due to the fact that Do et al. (2015) did not shift their spectra to the local standard of rest, as we did. This is a shift of less than 2 , and should therefore not affect the results severely (see Section 4.7).

\subsection{Note of caution on the measurement of high metallicities $[M / H] \gtrsim+\mathbf{0 . 5} \mathrm{dex}$}

Our results suggest that most stars are metal-rich, i.e., $[M / H]>0.0$ dex. For more than 75 per cent of the stars we obtain a metallicity $[M / H]>0.0 \mathrm{dex}$, and for about 28 per cent even $[M / H]>+0.5$ dex. The measurements of high metallicities have to be regarded with caution. Our metallicity measurements are differential and may not be correct in absolute values.

As Do et al. (2015) pointed out, there might be systematic effects due to the use of medium-resolution spectroscopy. Differences between metal-rich and solar-metallicity spectra are not as pronounced as differences between metal-poor and solar-metallicity spectra. We showed in Section 3.2.2 on the fit of a reference star that the metallicity can be overestimated by almost 0.7 dex. Fig. 3 illustrates that several lines of the top spectrum (black) are deeper than the lines of the best-fitting model spectrum (red). The model spectrum with the reference stellar paramters (blue) of Smith (1984) is a worse fit. A similar effect can be seen in the KMOS data. In Fig. 1, we showed the spectrum of a star with supposedly high metallicity $([M / H]=+0.69 \mathrm{dex}$, bottom spectrum). Although the model spectrum (red) has a high metallicity, several lines of the observed spectrum (black) are still deeper and not fitted well. It might be that the used model spectra are not ideal to fit red giants with high metallicities at medium spectral resolution. Maybe the assumption of solar $\alpha$-abundances causes problems at high metallicities. Alternatively, the line strengths of strong lines in the model spectra may be too low if the microturbulence assumed by Husser et al. (2013) was too low. Further, the synthetic spectra were computed assuming local thermodynamic equilibrium (LTE), which may affect our results (Lind et al. 2012; Bergemann \& Nordlander 2014). However, Lind et al. (2012) showed that non-LTE effects are most important for extremely metal-poor stars $([\mathrm{Fe} / \mathrm{H}]<-3 \mathrm{dex})$ and 
hot $\left(T_{\text {eff }}>5000 \mathrm{~K}\right)$ giants, and can lead to an underestimation of the Fe abundance from optical spectra. If the same is true for metallicities in the near-infrared, our results for the cool, metal-rich giants are unaffected by non-LTE effects. The Gaia-ESO Survey and the APOGEE measured metallicities for several thousand stars of the Milky Way (Mikolaitis et al. 2014; Holtzman et al. 2015). They found some stars with high metallicities $[M / H] \approx+0.5$ dex. However, their spectra are at shorter wavelengths than our data, and we cannot compare their spectra of metal-rich stars to our data. In summary, we cannot rule out that some of the stars in our data set have high metallicities. But since the spectra of the alleged super-metal-rich stars are not fitted well, we do not claim the detection of such stars.

Nevertheless, we conclude that most of the stars in our data set are metal-rich $([M / H]>+0.0 \mathrm{dex})$. Studies of the Milky Way bulge and disc showed that the metallicity increases towards the centre (e.g. Zoccali et al. 2008; Hayden et al. 2015; Ness \& Freeman 2016), with the most metalrich stars concentrated to the plane. Therefore, it is not surprising to find metal-rich stars in the Galactic Centre. To verify the metallicity measurements, observations with higher spectral resolution are required. A subsample of the metal-poor stars and some metal-rich stars observed with high-resolution spectroscopy would be useful to calibrate our measurements, and to measure abundance ratios, such as $[\mathrm{Na} / \mathrm{Fe}]$, and $[\mathrm{Ca} / \mathrm{Fe}]$. But also the applied tools should be improved. As the Galactic Centre stars seem to be enhanced in some elements, synthetic spectra with higher element abundances are probably more suitable to fit the spectra. At the moment, PHOENIX spectra with $[\alpha / F e]>0$ dex are only available for $[M / H]=0.0$ dex. We plan to implement different $\alpha$-abundances to the spectral fit in the future. In addition, a larger spectral library in the $K$ band at high to medium spectral resolution will be useful. In the near future, the X-SHOOTER spectral library (Chen et al. 2014) will provide near-infrared spectra, which will be very useful to study the Milky Way nuclear star cluster.

\subsection{Metallicity distribution}

The metallicity distribution is important to derive the fraction of metal-poor and metal-rich stars. This information is essential to reconstruct the star formation history. We obtained a mean metallicity $\langle[M / H]\rangle=+0.26$ dex. The standard deviation of $[M / H]$ is 0.42 dex.

Other studies obtained similar results for the metallicity distribution in the Galactic Centre. Do et al. (2015) found a mean value of $\langle[\mathrm{M} / \mathrm{H}]\rangle=+0.4$ dex and a standard deviation of 0.4 dex for a sample of 83 stars. Further out, in the inner Galactic bulge, Schultheis et al. (2015) measured the metallicities of 33 stars and obtained a mean metallicity $\langle[M / H]\rangle=+0.4$ dex with a dispersion of 0.55 dex. They also found eight low-metallicity stars with $[M / H] \approx-1.0$ dex and enhanced $\alpha$-element abundance. Our metallicity measurements are in agreement. Due to the larger number of stars in our sample, we were able to find a continuous metallicity distribution. The metallicity distribution is rather smooth, and we do not see signs of a second peak at $[M / H]=-1.0 \mathrm{dex}$, as indicated by the smaller samples of Do et al. (2015) and Schultheis et al. (2015). However, a smaller second metallicity peak may be undetectable given our measurement uncertainties. Our metallicity distribution has a negative skewness, which was also found for the metallicity distribution of the inner Galactic disc using APOGEE data (Hayden et al. 2015). However, we note that we did not measure systematic uncertainties for metallicities $[M / H]<-0.5$ dex and $[M / H]>+0.3$ dex on reference stars, but assumed the same systematic uncertainties as in the range $-0.5 \operatorname{dex}<[M / H]<+0.3$ dex. The shape of the metallicity distribution at low $([M / H]<-0.5$ dex $)$ and high $([M / H]>0.3 \mathrm{dex})$ metallicities may be influenced by unknown systematic effects. Nevertheless, the spread of the metallicity distribution by about 0.4 dex means that the chemical composition of the stars in the Galactic Centre is inhomogeneous.

\subsection{Contamination from foreground or background sources}

We checked the membership of a star to the Milky Way nuclear star cluster based on the extinction corrected colours. We identified foreground stars, e.g., from the Galactic bulge or disc, with their blue colours. But as we do not know exactly the extinction along the line of sight, it is possible that some stars of our sample are bar or bulge stars with rather high extinction. However, their number density should be lower than the number density of Galactic Centre stars. Clarkson et al. (2012) studied a $12 \operatorname{arcsec} \times 12 \operatorname{arcsec}$ field near the Arches cluster, about $26 \mathrm{pc}$ in projection away from our field. Their data contain only one field star with $K<13.8 \mathrm{mag}$, identified by proper motions. This translates to a number density of 0.007 inner bulge stars per $\operatorname{arcsec}^{2}$. In our field of $2700 \mathrm{arcsec}^{2}$, we estimate the number of inner bulge stars to approximately 19, but this estimate is based on a small field with only one foreground star in our magnitude range.

We also checked if the kinematics of the stars contain any hints on the cluster membership. For this reason, we matched the stars in our data set with the proper motion data of Schödel et al. (2009). We obtained 400 matches, all within the central $p=25 \operatorname{arcsec}(\sim 0.97 \mathrm{pc})$ of the nuclear star cluster. Most of the stars have a lower velocity than the escape velocity, and their kinematics are in agreement with being bound to the nuclear star cluster. There is one exception with a significantly higher velocity than the escape velocity, this is the potential runaway star found by Schödel et al. (2009). It has a proper motion velocity of $424 \mathrm{~km} \mathrm{~s}^{-1}$. Schödel et al. (2009) suggested that this star might escape the Milky Way nuclear star cluster. About 40 per cent of the stars move against the main direction of rotation of the nuclear star cluster. Several stars in the Galactic East have a negative velocity $v_{z}$, and likewise, several stars in the Galactic West have a positive velocity. But this does not mean that these stars do not belong to the nuclear star cluster. They may be on bound counter-rotating tube orbits or on box orbits.

There might be some stars from the nuclear stellar disc with similar colours as the stars of the nuclear star cluster. The nuclear stellar disc and the nuclear star cluster form the nuclear stellar bulge. The nuclear stellar disc extends over $p \approx 120 \mathrm{pc}$, and dominates over the nuclear star cluster at $p \gtrsim 30 \mathrm{pc}$ (Launhardt et al. 2002). We are not able to 
distinguish if a star is from the nuclear star cluster or the nuclear stellar disc based on the extinction or kinematics.

\subsection{Luminosity classes and implications for stellar ages}

The $K_{S}$-band magnitude can be used to infer the luminosity class of the stars, and estimate their age. The extinction corrected magnitudes of the stars in our data set range from $K_{S, 0}<6$ to $K_{S, 0} \approx 13.5 \mathrm{mag}$, with a median at $K_{S, 0}=10.4 \mathrm{mag}$.

Stars with magnitudes $K_{S, 0} \lesssim 5.7 \mathrm{mag}$ are red supergiants (Blum et al. 2003), but supergiants may be as faint as approximately $7.5 \mathrm{mag}$ (Blum et al. 1996, 2003). Red supergiants are massive $\left(\mathcal{M}>10 \mathrm{M}_{\odot} ;\right.$ Cox 2000$)$ and young, only a few $10^{7}$ yr old (Greggio \& Renzini 2011). For five stars in the range $5.7 \mathrm{mag} \lesssim K_{S, 0} \lesssim 7.5 \mathrm{mag}$, one cannot distinguish supergiants from (bright) giant stars based on the magnitude. Blum et al. (2003) suggested that it is more likely for the stars to be (bright) giants.

Red giant stars have masses of about $0.3-10 \mathrm{M}_{\odot}$, and ages $\gtrsim 1$ Gyr. The stars with $K_{S, 0}>7.5 \mathrm{mag}$ are probably red giant stars. This means 99 per cent of the stars in our data set are red giants. The red clump is a subgroup of red giants, their magnitude is roughly $K_{S, 0}=12.9 \mathrm{mag}$ (Schödel et al. 2010). Only about 1.5 per cent of the stars in our data set are faint enough for the red clump. Another subgroup of red giant stars are the bright asymptotic giant branch (AGB) stars. AGB stars have masses in the range $0.5 \mathrm{M}_{\odot} \lesssim \mathcal{M} \lesssim 10 \mathrm{M}_{\odot}$ (Blum et al. 1996). They have two phases, the early AGB (E-AGB) phase, and a later thermally pulsing AGB (TP-AGB) phase. During the later phase, material from the core is mixed with the surface material. The six long-period variables (Section 4.5) are probably such AGB stars. TP-AGB stars are rather bright, with $K_{S, 0} \lesssim 8.4$ mag for solar and higher metallicities and ages $>1$ Gyr (PARSEC evolutionary tracks; Bressan et al. 2012; Rosenfield et al. 2016). About 50 stars (7 per cent) in our sample could be TP-AGB stars, based on their $K_{S}$-band magnitudes. Fainter stars could be E-AGB stars (6.9 mag $\lesssim K_{S, 0} \lesssim 12 \mathrm{mag}, 93$ per cent) or normal red giant branch stars $\left(7.5 \mathrm{mag} \lesssim K_{S, 0} \lesssim 16.4 \mathrm{mag}, 99\right.$ per cent). As the time a star spends on the red giant branch is about 40 times longer than the time spent as AGB star (Greggio \& Renzini 2011), it is more likely for a star to be on the red giant branch.

The metal-poor stars in our data set have magnitudes ranging from $K_{S, 0}=7.15$ to $12.56 \mathrm{mag}$ and temperatures from 3660 to $5300 \mathrm{~K}$, except for one cooler long-period variable star. We compared the location of the stars in a $T_{\text {eff }}^{-}$ $K_{S, 0}$ diagram with the PARSEC evolutionary tracks (Bressan et al. 2012). Using $[M / H]=-0.5$ and -1.0 dex for the tracks, we find that the metal-poor stars have ages $\gtrsim 1 \mathrm{Gyr}$, and initial masses $\mathcal{M}_{\text {ini }} \lesssim 2 \mathrm{M}_{\odot}$. Tracks with age $10 \mathrm{Gyr}$ and masses $\mathcal{M}_{\text {ini }} \lesssim 1 \mathrm{M}_{\odot}$ are also in agreement with the stellar effective temperatures and magnitudes. This means the stellar ages and masses are consistent with stars in Galactic globular clusters.

\subsection{Clues to the formation of the Milky Way nuclear star cluster}

There is a large spread of metallicities ranging from metal-poor stars $([M / H] \lesssim-1.0 \mathrm{dex})$ to metal-rich stars $([M / H] \gtrsim+0.2 \mathrm{dex})$ in the late-type giant star population of the Milky Way nuclear star cluster. This means that the cluster did not form from one homogeneous gas cloud in one single starburst.

The metal-poor stars in the Milky Way nuclear star cluster may have formed in an early starburst in situ, or somewhere else in the Galaxy, and migrated to the centre. The stars might have migrated from the Milky Way bulge to the centre, as the inner bulge also contains some metalpoor stars (Schultheis et al. 2015; Ness \& Freeman 2016). Another possibility is that the stars formed in a star cluster, potentially a globular cluster, that migrated to the centre of the Milky Way. The Milky Way globular cluster system has a bimodal metallicity distribution, with about 71 per cent metal-poor $([\mathrm{Fe} / \mathrm{H}] \lesssim-0.75 \mathrm{dex})$ and 29 per cent metal-rich $(-0.75 \lesssim[\mathrm{Fe} / \mathrm{H}] \lesssim 0.0$ dex $)$ globular clusters (Bica et al. 2006). The subsolar metallicity stars in the Milky Way nuclear star cluster have similar metallicities as the "metalrich' Milky Way globular clusters.

A large fraction of stars in the Milky Way nuclear star cluster is metal-rich $([M / H]>0.0 \mathrm{dex})$. These stars cannot originate from the infall of globular clusters to the centre of the Milky Way. The metal-rich stars must have formed from enriched gas within the Milky Way, as their metallicities are inconsistent with the observed Milky Way globular cluster metallicities. Metal-rich stars formed either directly in the Galactic Centre, or in an enriched star cluster within the Milky Way that migrated to the Galactic Centre.

The spatial distribution and stellar kinematics should be able to discern the in situ formation and cluster infall scenario for the different stellar populations. Aharon \& Perets (2015) simulated the in situ formation scenario for nuclear star clusters. Depending on where the star formation occurs dominantly, in the centre or in the outskirts of the nuclear star cluster, the cluster would have a positive or negative age gradient. But also the consecutive infall of multiple stellar clusters creates an age and stellar population gradient in the nuclear star cluster (Perets \& Mastrobuono-Battisti 2014). We investigated the radial distribution of metal-poor stars, but we were not able to find any significant difference with respect to metal-rich stars. The number of metal-poor stars is too low, and the uncertainties are too high to discern the spatial distributions. We found indications for a slight overabundance of metal-poor stars in the Galactic North East. If this observation is confirmed, it would rather suggest a later infall scenario than in situ formation. If the old, metal-poor stars have formed in situ, they should be distributed isotropically now. However, this assumption needs to be confirmed in three-dimensional simulations of in situ nuclear star cluster formation. The kinematics of the metal-poor stars are not significantly different from the kinematics of the other stars. The dynamical evolution of the different formation scenarios needs to be studied further in simulations. 


\section{CONCLUSIONS}

We observed the central $4 \mathrm{pc}^{2}$ of the Milky Way nuclear star cluster in the $K$ band with the integral-field spectrograph KMOS. We analysed the spectra of more than 700 late-type stars. We found that the equivalent width of the Na I doublet line region is enhanced with respect to stellar library spectra. Using full-spectral fitting, we derived effective temperatures and metallicities of 705 stars of the Milky Way nuclear star cluster. Most stars are red K- and M-type giants with temperatures $T_{\text {eff }}=3000-5000 \mathrm{~K}$. The metallicities range from subsolar $[M / H]<-1.0$ dex to supersolar $[M / H]>+0.3 \mathrm{dex}$, with a standard deviation of $0.42 \mathrm{dex}$. This large metallicity spread rules out a scenario in which the nuclear star cluster formed in a single burst of star formation. The fraction of low-metallicity stars with $[M / H] \leqslant-0.5$ dex is $\left(5.2_{-3.1}^{+6.0}\right)$ per cent. The spatial distributions and kinematics of metal-poor stars and metal-rich stars are similar. The metal-poor stars might originate from infalling globular clusters. However, our data set is dominated by metal-rich stars, and these stars have higher metallicities than the most metal-rich Milky Way globular clusters. A scenario in which the nuclear star cluster is entirely formed from infalling globular clusters can be ruled out.

\section{ACKNOWLEDGEMENTS}

RS acknowledges funding from the European Research Council under the European Union's Seventh Framework Programme (FP7/2007-2013) / ERC grant agreement no. 614922. This publication makes use of data products from the 2MASS, which is a joint project of the University of Massachusetts and the Infrared Processing and Analysis Center/California Institute of Technology, funded by the National Aeronautics and Space Administration and the National Science Foundation. This research made use of the SIMBAD data base (operated at CDS, Strasbourg, France). We thank Nikolay Kacharov, and Iskren Georgiev for discussions and suggestions. Thanks to Ariane Lançon for helpful discussions. We finally thank the anonymous referee for comments and suggestions. Based on observations collected at the European Organisation for Astronomical Research in the Southern Hemisphere, Chile (60.A-9450(A)).

\section{REFERENCES}

Agarwal M., Milosavljević M., 2011, ApJ, 729, 35

Aharon D., Perets H. B., 2015, ApJ, 799, 185

Antonini F., 2013, ApJ, 763, 62

Antonini F., Capuzzo-Dolcetta R., Mastrobuono-Battisti A., Merritt D., 2012, ApJ, 750, 111

Bergemann M., Nordlander T., 2014, NLTE Radiative Transfer in Cool Stars, Niemczura E., Smalley B., Pych W., eds., Springer International Publishing, pp. 169-185

Bica E., Bonatto C., Barbuy B., Ortolani S., 2006, A\&A, 450, 105

Blum R. D., Ramírez S. V., Sellgren K., Olsen K., 2003, ApJ, 597,323

Blum R. D., Sellgren K., Depoy D. L., 1996, AJ, 112, 1988

Böker T., Laine S., van der Marel R. P., Sarzi M., Rix H.-W., Ho L. C., Shields J. C., 2002, AJ, 123, 1389
Bressan A., Marigo P., Girardi L., Salasnich B., Dal Cero C., Rubele S., Nanni A., 2012, MNRAS, 427, 127

Buchner J., Georgakakis A., Nandra K., Hsu L., Rangel C., Brightman M., Merloni A., Salvato M. et al, 2014, A\&A, 564, A125

Cappellari M., Emsellem E., 2004, PASP, 116, 138

Capuzzo-Dolcetta R., Miocchi P., 2008, ApJ, 681, 1136

Chatzopoulos S., Fritz T. K., Gerhard O., Gillessen S., Wegg C., Genzel R., Pfuhl O., 2015, MNRAS, 447, 948

Chen Y.-P., Trager S. C., Peletier R. F., Lançon A., Vazdekis A., Prugniel P., Silva D. R., Gonneau A., 2014, A\&A, 565, A117

Clarkson W. I., Ghez A. M., Morris M. R., Lu J. R., Stolte A., McCrady N., Do T., Yelda S., 2012, ApJ, 751, 132

Clénet Y., Rouan D., Gendron E., Montri J., Rigaut F., Léna P., Lacombe F., 2001, A\&A, 376, 124

Côté P., Piatek S., Ferrarese L., Jordán A., Merritt D., Peng E. W., Haşegan M., Blakeslee J. P. et al, 2006, ApJS, 165, 57

Cox A. N., ed., 2000, Allen's Astrophysical Quantities, 4th edn. AIP Press, New York

Cunha K., Sellgren K., Smith V. V., Ramirez S. V., Blum R. D., Terndrup D. M., 2007, ApJ, 669, 1011

Davies R. I., 2007, MNRAS, 375, 1099

Do T., Kerzendorf W., Winsor N., Støstad M., Morris M. R., Lu J. R., Ghez A. M., 2015, ApJ, 809, 143

Do T., Lu J. R., Ghez A. M., Morris M. R., Yelda S., Martinez G. D., Wright S. A., Matthews K., 2013, ApJ, 764, 154

Dutra C. M., Bica E., 2001, A\&A, 376, 434

Feldmeier A., Neumayer N., Seth A., Schödel R., Lützgendorf N., de Zeeuw P. T., Kissler-Patig M., Nishiyama S. et al, 2014, A\&A, 570, A2

Feldmeier-Krause A., Neumayer N., Schödel R., Seth A., Hilker M., de Zeeuw P. T., Kuntschner H., Walcher C. J. et al, 2015, A\&A, 584, A2

Feroz F., Hobson M. P., Bridges M., 2009, MNRAS, 398, 1601

Frogel J. A., Stephens A., Ramírez S., DePoy D. L., 2001, AJ, 122,1896

Gazak J. Z., Kudritzki R., Evans C., Patrick L., Davies B., Bergemann M., Plez B., Bresolin F. et al, 2015, ApJ, 805, 182

Ghez A. M., Salim S., Weinberg N. N., Lu J. R., Do T., Dunn J. K., Matthews K., Morris M. R. et al, 2008, ApJ, 689, 1044

Gillessen S., Eisenhauer F., Fritz T. K., Bartko H., Dodds-Eden K., Pfuhl O., Ott T., Genzel R., 2009, ApJ, 707, L114

Gnedin O. Y., Ostriker J. P., Tremaine S., 2014, ApJ, 785, 71

Graham A. W., Spitler L. R., 2009, MNRAS, 397, 2148

Gray D. F., 1989, ApJ, 347, 1021

Greggio L., Renzini A., 2011, Stellar Populations: A User Guide from Low to High Redshift. Wiley, New York

Gustafsson B., Edvardsson B., Eriksson K., Jørgensen U. G., Nordlund A., Plez B., 2008, A\&A, 486, 951

Harris W. E., 1996, AJ, 112, 1487

Hayden M. R., Bovy J., Holtzman J. A., Nidever D. L., Bird J. C., Weinberg D. H., Andrews B. H., Majewski S. R. et al, 2015, ApJ, 808, 132

Holtzman J. A., Shetrone M., Johnson J. A., Allende Prieto C., Anders F., Andrews B., Beers T. C., Bizyaev D. et al, 2015, AJ, 150, 148

Houdashelt M. L., Frogel J. A., Cohen J. G., 1992, AJ, 103, 163 Husser T.-O., Wende-von Berg S., Dreizler S., Homeier D., Reiners A., Barman T., Hauschildt P. H., 2013, A\&A, 553, A6

Ivanov V. D., Rieke M. J., Engelbracht C. W., Alonso-Herrero A., Rieke G. H., Luhman K. L., 2004, ApJS, 151, 387

Kamann S., Wisotzki L., Roth M. M., 2013, A\&A, 549, A71

Karakas A. I., 2010, MNRAS, 403, 1413

Kerzendorf W., Do T., 2015, starkit: First real release

Kobayashi C., Umeda H., Nomoto K., Tominaga N., Ohkubo T., 2006, ApJ, 653, 1145

Lang K. R., 1992, Astrophysical data I: Planets and stars. Springer-Verlag, Berlin 
Launhardt R., Zylka R., Mezger P. G., 2002, A\&A, 384, 112

Lind K., Bergemann M., Asplund M., 2012, MNRAS, 427, 50

Loose H. H., Kruegel E., Tutukov A., 1982, A\&A, 105, 342

Mármol-Queraltó E., Cardiel N., Cenarro A. J., Vazdekis A., Gorgas J., Pedraz S., Peletier R. F., Sánchez-Blázquez P., 2008, A\&A, 489, 885

Matsunaga N., Kawadu T., Nishiyama S., Nagayama T., Hatano H., Tamura M., Glass I. S., Nagata T., 2009, MNRAS, 399, 1709

McGinn M. T., Sellgren K., Becklin E. E., Hall D. N. B., 1989, ApJ, 338, 824

McWilliam A., 1990, ApJS, 74, 1075

Mikolaitis Š., Hill V., Recio-Blanco A., de Laverny P., Allende Prieto C., Kordopatis G., Tautvaišiene G., Romano D. et al, 2014, A\&A, 572, A33

Neeser M., Coccato L., Jung Y., Freudling W., 2016, Reflex KMOS Tutorial Issue 1.6

Ness M., Freeman K., 2016, Publ. Astron. Soc. Australia, 33, e022 Neumayer N., Walcher C. J., 2012, Adv. Astron., 2012, 709038

Nishiyama S., Nagata T., Kusakabe N., Matsunaga N., Naoi T., Kato D., Nagashima C., Sugitani K. et al, 2006, ApJ, 638, 839

Nishiyama S., Schödel R., Yoshikawa T., Nagata T., Minowa Y., Tamura M., 2016, A\&A, 588, A49

Oke J. B., Greenstein J. L., 1954, ApJ, 120, 384

Perets H. B., Mastrobuono-Battisti A., 2014, ApJ, 784, L44

Pflamm-Altenburg J., Kroupa P., 2009, MNRAS, 397, 488

Pfuhl O., Fritz T. K., Zilka M., Maness H., Eisenhauer F., Genzel R., Gillessen S., Ott T. et al, 2011, ApJ, 741, 108

Pryor C., Meylan G., 1993, in ASP Conf. Ser., Vol. 50, Structure and Dynamics of Globular Clusters, Djorgovski S. G., Meylan G., eds., Astron. Soc. Pac., San Francisco, p. 357

Ramírez S. V., Sellgren K., Carr J. S., Balachandran S. C., Blum R., Terndrup D. M., Steed A., 2000, ApJ, 537, 205

Rayner J. T., Cushing M. C., Vacca W. D., 2009, ApJS, 185, 289

Reid M. J., Menten K. M., Genzel R., Ott T., Schödel R., Eckart A., 2003, ApJ, 587, 208

Rosenfield P., Marigo P., Girardi L., Dalcanton J. J., Bressan A., Williams B. F., Dolphin A., 2016, ApJ, 822, 73

Ryde N., Schultheis M., 2015, A\&A, 573, A14

Ryde N., Schultheis M., Grieco V., Matteucci F., Rich R. M., Uttenthaler S., 2016, AJ, 151, 1

Schinnerer E., Böker T., Meier D. S., Calzetti D., 2008, ApJ, 684, L21

Schödel R., Feldmeier A., Kunneriath D., Stolovy S., Neumayer N., Amaro-Seoane P., Nishiyama S., 2014a, A\&A, 566, A47

Schödel R., Feldmeier A., Neumayer N., Meyer L., Yelda S., 2014b, Class. Quantum Grav., 31, 244007

Schödel R., Merritt D., Eckart A., 2009, A\&A, 502, 91

Schödel R., Najarro F., Muzic K., Eckart A., 2010, A\&A, 511, A18

Schultheis M., Cunha K., Zasowski G., García Pérez A. E., Sellgren K., Smith V., García-Hernández D. A., Zamora O. et al, 2015, A\&A, 584, A45

Schultheis M., Ryde N., Nandakumar G., 2016, A\&A, 590, A6

Scoville N. Z., Stolovy S. R., Rieke M., Christopher M., YusefZadeh F., 2003, ApJ, 594, 294

Seth A., Agüeros M., Lee D., Basu-Zych A., 2008a, ApJ, 678, 116

Seth A. C., Blum R. D., Bastian N., Caldwell N., Debattista V. P., 2008b, ApJ, 687, 997

Sharples R., Bender R., Agudo Berbel A., Bezawada N., Castillo R., Cirasuolo M., Davidson G., Davies R. et al, 2013, The Messenger, 151, 21

Silva D. R., Kuntschner H., Lyubenova M., 2008, ApJ, 674, 194

Skrutskie M. F., Cutri R. M., Stiening R., Weinberg M. D., Schneider S., Carpenter J. M., Beichman C., Capps R. et al, 2006, AJ, 131, 1163

Smith V. V., 1984, A\&A, 132, 326
Soubiran C., Le Campion J.-F., Cayrel de Strobel G., Caillo A., 2010, A\&A, 515, A111

Tremaine S. D., Ostriker J. P., Spitzer Jr. L., 1975, ApJ, 196, 407 van Dokkum P. G., 2001, PASP, 113, 1420

Wallace L., Hinkle K., 1996, ApJS, 107, 312

—, 1997, ApJS, 111, 445

Wenger M., Ochsenbein F., Egret D., Dubois P., Bonnarel F., Borde S., Genova F., Jasniewicz G. et al, 2000, A\&AS, 143, 9

Winge C., Riffel R. A., Storchi-Bergmann T., 2009, ApJS, 185, 186

Zoccali M., Hill V., Lecureur A., Barbuy B., Renzini A., Minniti D., Gómez A., Ortolani S., 2008, A\&A, 486, 177

\section{APPENDIX A: THE $T_{\text {eff }}-E W_{\text {CO }}$ RELATION FOR GIANTS}

We calibrated the relation of the effective temperature $T_{\text {eff }}$ with the $\mathrm{CO}$ equivalent width $E W_{\mathrm{CO}}$ as defined by Frogel et al. (2001) using the stars of the spectral library. We considered 69 stars with luminosity classes II-IV. EW $W_{\mathrm{CO}}$ was computed with $R=3000$ for stars from Wallace \& Hinkle (1997), and $R=4350$ for the other stars in the spectral library.

The effective temperature $T_{\text {eff }}$ is given for the stars in the GNIRS library in Winge et al. (2009), for the other stars we complemented $T_{\text {eff }}$ with entries from the stellar parameter catalogue PASTEL (Soubiran et al. 2010). For stars with more than one entry we used the mean of the various measurements, and the standard deviation as uncertainty $\sigma_{T_{\text {eff }} \text {. }}$. When there was no entry of $T_{\text {eff }}$ for a star in the PASTEL catalogue, we used our knowledge of the spectral type. We used the list given in Lang (1992), where $T_{\text {eff }}$ is listed for various spectral types, and for dwarfs, giants, and supergiants separately. When necessary, we interpolated linearly between the spectral classes. We compared the Lang (1992) values for $T_{\text {eff }}$ with the values given in tables $7.5,7.6$ and 7.7 in Cox (2000) and in table 15.7 in Cox (2000). We used the scatter between these three different spectral type- $T_{\text {eff }}$ tables to estimate the uncertainty $\sigma_{T_{\text {eff }}}$.

Fig. A1 shows the relation between $T_{\text {eff }}$ and $E W_{\mathrm{CO}}$ for 69 stars with luminosity classes II-IV from the spectral libraries. We made a linear fit to the data in the range below $6000 \mathrm{~K}$ and found the relation

$T_{\text {eff }}=5677^{ \pm 21} \mathrm{~K}-106.3^{ \pm 3.0} \mathrm{~K}^{-1} \times E W_{\mathrm{CO}}$,

where $E W_{\mathrm{CO}}$ is in, and $T_{\text {eff }}$ in $\mathrm{K}$. The uncertainties are the formal fit uncertainties. The residual scatter is $163 \mathrm{~K}$. Our best-fitting result is shown as red line in Fig. A1. We also show the three-degree-polynomial fit of Pfuhl et al. (2011) as blue dot-dashed line. Pfuhl et al. (2011) used 33 giant stars from spectral libraries at lower spectral resolution $(R \approx 3000$ and $\approx 2000)$. Our $T_{\text {eff }}-E W_{\mathrm{CO}}$ relation is in agreement with their relation for $T_{\text {eff }} \gtrsim 3000 \mathrm{~K}$. However, at $T_{\text {eff }} \lesssim 3000 \mathrm{~K}$, the Pfuhl et al. (2011) relation declines faster than our linear fit. The different result of the relation can be explained by the larger sample and the different values of $T_{\text {eff }}$ that we use. We included larger uncertainties $\sigma_{T_{\text {eff }}}$ than Pfuhl et al. (2011), since we took several measurements of $T_{\text {eff }}$ for a star into account. We did not make a metallicity cut for the stars, since for many stars in the stellar library the metallicity is not known. When we just use a subsample of 39 spectra with near solar-metallicity in 


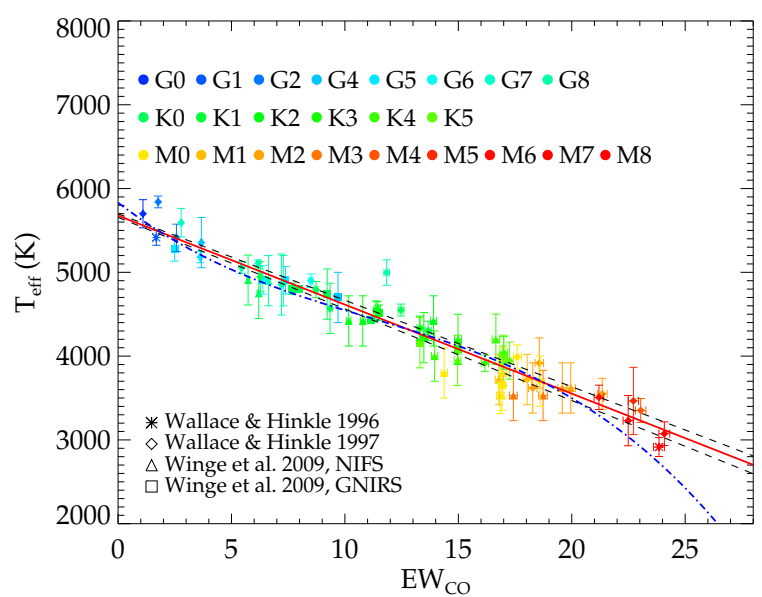

Figure A1. Relation between effective temperature $T_{\text {eff }}$ and the equivalent width $E W_{\mathrm{CO}}$ for 69 template stars with luminosity class II-IV. The different colours denote different spectral types, the symbols denote the library from which the spectrum is taken. The red line is a linear fit to the data, the black dashed lines show the formal uncertainty of the linear fit. The blue dot-dashed line is the $T_{\mathrm{eff}}-E W_{\mathrm{CO}}$ relation as derived by Pfuhl et al. (2011).

the range $-0.5 \operatorname{dex}<[M / H]<+0.5$ dex, the relation changes within the formal fit uncertainties. Schultheis et al. (2016) showed that the $T_{\text {eff }}-E W_{\mathrm{CO}}$ relation does not depend on the metallicity in the temperature range $3200-4500 \mathrm{~K}$ and metallicities $[\mathrm{Fe} / \mathrm{H}]$ from -1.2 to +0.5 dex. We conclude that our $T_{\text {eff }}-E W_{\mathrm{CO}}$ relation is robust in this temperature and metallicity range.

\section{APPENDIX B: TABLE OF STELLAR \\ PARAMETERS}


Table B1. Stellar parameters: stellar identification number Id, the coordinates in RA and Dec., the stellar parameters $T_{\text {eff }},[M / H]$, $\log (g), v_{z}$, and extinction corrected $K_{S}$-band magnitude $K_{S, 0}$. The full table is available online.

\begin{tabular}{ccccccccccc} 
Id & $\begin{array}{c}\text { RA } \\
\left({ }^{\circ}\right.\end{array}$ & $\begin{array}{c}\text { Dec. } \\
\left({ }^{\circ}\right)\end{array}$ & $\begin{array}{c}T_{\text {eff }} \\
(\mathrm{K})\end{array}$ & $\begin{array}{c}\sigma_{T_{\text {eff }}}(\mathrm{K}) \\
{[1}\end{array}$ & $\begin{array}{c}{[M / H]} \\
(\mathrm{dex})\end{array}$ & $\begin{array}{c}\sigma_{[M / H]} \\
(\mathrm{dex})\end{array}$ & $\begin{array}{c}\log (g) \\
(\mathrm{dex})\end{array}$ & $\begin{array}{c}\sigma_{\log (g)} \\
(\operatorname{dex})\end{array}$ & $\begin{array}{c}v_{z} \\
\left(\mathrm{~km} \mathrm{~s}^{-1}\right)\end{array}$ & $\begin{array}{c}K_{S, 0} \\
(\mathrm{mag})\end{array}$ \\
\hline 5 & 266.41675 & -29.010296 & 3190 & 209 & 0.87 & 0.31 & 1.2 & 1.1 & 25.6 & 7.44 \\
6 & 266.41571 & -29.012167 & 3301 & 206 & 0.13 & 0.25 & 0.0 & 1.0 & -241.4 & 7.88 \\
14 & 266.42401 & -29.003611 & 3374 & 205 & 0.14 & 0.25 & 0.0 & 1.0 & 133.5 & 7.79 \\
16 & 266.41727 & -29.004204 & 3250 & 217 & 0.31 & 0.24 & 0.1 & 1.0 & 69.5 & 7.78 \\
17 & 266.42236 & -29.0062938 & 3200 & 205 & 0.31 & 0.25 & 0.0 & 1.0 & -65.2 & 7.71 \\
\hline
\end{tabular}

This paper has been typeset from a $\mathrm{T}_{\mathrm{E}} \mathrm{X} / \mathrm{L} \mathrm{T} \mathrm{E} \mathrm{X}$ file prepared by the author. 